Pacific

Journal of

Mathematics

\title{
ON THE SET OF MAXIMAL NILPOTENT SUPPORTS OF SUPERCUSPIDAL REPRESENTATIONS
}

QIN YUJUN 


\title{
ON THE SET OF MAXIMAL NILPOTENT SUPPORTS OF SUPERCUSPIDAL REPRESENTATIONS
}

\author{
QIN YUJUN
}

\begin{abstract}
Let $G$ be a quasisplit reductive group over a $p$-adic field $k, T$ a maximal unramified anisotropic torus of $G(k)$, and $\chi$ a character of $T(k)$ satisfying certain conditions. Assume the residue characteristic $p$ of $k$ is large enough. It was shown by DeBacker and Reeder that the irreducible supercuspidal representation $\pi_{\chi}$ of $G(k)$ associated to $(T(k), \chi)$ is generic if and only if $\mathscr{B}(T, k)$ is a special vertex of $\mathscr{B}(G, k)$. We compute the set of maximal nilpotent support $\mathcal{N}_{\text {wh,max }}\left(\pi_{\chi}\right)$ when $\mathscr{B}(T, k)$ is not a special point in $\mathscr{B}(G, k)$.
\end{abstract}

\section{Introduction}

Let $k$ be a $p$-adic field and $\psi$ a nontrivial character of $k$. Let $\boldsymbol{G}$ be a split orthogonal or symplectic group over $k, \mathfrak{g}$ the Lie algebra of $\boldsymbol{G}, G=\boldsymbol{G}(k)$, and $\mathfrak{g}=\mathfrak{g}(k)$. Let $\mathfrak{g}_{\text {nil }}$ be the set of nilpotent elements in $\mathfrak{g}$ upon which $G$ acts by the adjoint action. Let $O$ be an orbit in $\mathfrak{g}_{\text {nil }} / G, z \in O$, and let $\phi: \mathfrak{s l}_{2} \rightarrow \mathfrak{g}$ be a Lie algebra homomorphism with

$$
\phi\left(\left(\begin{array}{ll}
0 & 0 \\
1 & 0
\end{array}\right)\right)=z
$$

Identify a scalar $t \in k$ with the diagonal matrix $\operatorname{diag}\left(t, t^{-1}\right) \in \mathfrak{s l}_{2}(k)$. For $j \in \mathbb{Z}$, let

$$
\mathfrak{g}_{j}=\{Y \in \mathfrak{g} \mid \operatorname{Ad} \circ \phi(t)(Y)=i t Y \text { for all } t \in k\} .
$$

Then $\mathfrak{g}$ has a decomposition $\mathfrak{g}=\bigoplus_{j \in \mathbb{Z}} \mathfrak{g}_{j}, z \in \mathfrak{g}_{-2}$.

Let $N_{\geq 2}$ (resp. $N_{\geq 1}$ ) be the unipotent subgroup of $G$ with Lie algebra $\mathfrak{n}_{\geq 2}=$ $\bigoplus_{j \geq 2} \mathfrak{g}_{j}\left(\right.$ resp. $\left.\mathfrak{n}_{\geq 1}=\bigoplus_{j \geq 1} \mathfrak{g}_{j}\right)$ and $\psi_{z}(n)=\psi(\operatorname{tr}(z \log n))$ be a character of $N_{\geq 2}$. Let $S_{z}$ be the irreducible representation of $N_{\geq 1}$ whose restriction to $N_{\geq 2}$ is a multiple of $\psi_{z}$. Let $\pi$ be an irreducible representation of $G$; following [Mœglin and Waldspurger 1987], let $\mathcal{N}_{\text {wh }}(\pi)$ be the subset of nilpotent orbits such that $O \in \mathcal{N}_{\text {wh }}(\pi)$ if and only if $\operatorname{Hom}_{N_{\geq 1}}\left(\pi, S_{z}\right) \neq 0$ for any $z \in O$. Let $\mathcal{N}_{\text {wh,max }}(\pi)$ be the subset of maximal elements in $\mathcal{N}_{\text {wh }}(\pi)$ with respect to the inclusion relation of closure of orbits.

MSC2010: 22E50.

Keywords: supercuspidal representations, Bruhat-Tits building, nilpotent orbits. 
On the other hand, let $\boldsymbol{T}$ be a maximal $K$-split anisotropic torus of $\boldsymbol{G}$; here, $K$ is the maximal unramified extension of $k$. Then $T=\boldsymbol{T}(k)$ is a maximal unramified anisotropic torus of $G$. Let $\chi$ be a character of $T$ satisfying certain conditions described in [Adler 1998] or [Reeder 2008]. There is a supercuspidal irreducible representation $\pi_{\chi}$ of $G$ associated to $(T, \chi)$. Identify $\mathscr{B}(\boldsymbol{T}, k)$ as a point in $\mathscr{B}(\boldsymbol{G}, k)$. In [DeBacker and Reeder 2010], it was shown that $\pi_{\chi}$ is generic (that is, $\mathcal{N}_{\text {wh }}\left(\pi_{\chi}\right)$ contains a regular nilpotent orbit) if and only if $\mathscr{B}(T, k)$ is a special point in $\mathscr{B}(G, k)$. In [Barbasch and Moy 1997], it was shown that if $\chi$ is of depth zero, the character of $\pi_{\chi}$ can be expanded as linear combination of orbital integrals over elements in $N_{\text {wh }}\left(\pi_{\chi}\right)$.

For those $(T, \chi)$ with $\mathscr{B}(\boldsymbol{T}, k)$ nonspecial (that is, when $\operatorname{rank}(G)$ is large enough for $\mathscr{B}(G)$ to contain nonspecial vertices), we show in Theorem 3.2 that if $\chi$ is of positive depth, there is one element in $\mathcal{N}_{\text {wh,max }}\left(\pi_{\chi}\right)$ which is related to $\mathscr{B}(\boldsymbol{T}, k)$. Note that in this case the supercuspidal representation $\pi_{\chi}$ is of positive integral depth. We also apply this theorem to irreducible representations in $\Pi_{\varphi}^{\prime}$, the $L$-packet of $\varphi$, where $\varphi$ is the Langlands parameter of $\pi_{\chi}$.

This article is organized as follows: in Section 2, preliminary notation are recalled, including vertices in Bruhat-Tits building, $L$-packet of positive-depth supercuspidal representations [Reeder 2008], classification of maximal unramified anisotropic tori [DeBacker 2006], and classification of rational nilpotent orbits [Waldspurger 2001]. We also show by example in the Appendix how to choose a particular element from a rational nilpotent orbit. The main theorems are stated and proved in Section 3.

\section{Preliminary}

2A. Notation. Let $k$ be a nonarchimedean local field of characteristic 0 with residue field $\mathfrak{f}$, and let $p$ be the characteristic of $\mathfrak{f}$. Let $\mathfrak{O}$ be the ring of integers of $k$ and $\mathfrak{P}$ the maximal ideal of $\mathfrak{O}$. Let $K$ be the maximal unramified field extension of $k$ and $\mathfrak{F}$ the residue field of $K$. Let $v$ be the normalized valuation of $k$ and $v_{K}$ the extension of $v$ to $K$. Let $\psi$ be an additive character of $k$ with conductor $\mathfrak{P}$, and denote the character of $\mathfrak{f}=\mathfrak{O} / \mathfrak{P}$ derived from $\psi$ by $\psi$ also.

Throughout this paper, assume $p$ is large enough that $p$ is a good prime in the sense in [Carter 1972].

Let $W$ be a finite-dimensional vector space over $k,\langle\cdot, \cdot\rangle$ a nondegenerate bilinear form on $W$, and $d=\operatorname{dim}_{k}(W)$. Assume that

$$
\langle v, w\rangle=\epsilon_{W}\langle w, v\rangle \quad \text { for all } v, w \in W,
$$

with $\epsilon_{W}= \pm 1$. Let $\boldsymbol{G}$ be the reductive group defined over $k$ with

$$
\boldsymbol{G}= \begin{cases}\mathbf{S O}(W) & \text { if } \epsilon_{W}=1 \\ \mathbf{S p}(W) & \text { if } \epsilon_{W}=-1\end{cases}
$$


Throughout this paper, assume that $W$ has a $k$-basis $\left\{e_{1}, \ldots, e_{d}\right\}$ satisfying

$$
\left\langle e_{j}, e_{k}\right\rangle= \begin{cases}0 & \text { if } j+k \neq d+1, \\ 1 & \text { if } j+k=d+1, j \leq k .\end{cases}
$$

Then $\boldsymbol{G}$ is a connected split reductive group over $k$ with finite center. Where no confusion will result, denote $\boldsymbol{G}$ by $\mathbf{S O}(d), \mathbf{S p}(d)$ for $\epsilon_{W}=1,-1$, respectively.

Let $J_{W}=\left(a_{i, j}\right)$ be the matrix of degree $d$ such that ${ }^{t} J_{W}=\epsilon_{W} J_{W}$ and

$$
a_{j, k}=\delta_{j, d+1-k} \text { for } j \leq k .
$$

Let $\bar{k}$ be the algebraic closure of $k$ and $R \subset \bar{k}$ a commutative $k$-algebra. Then $\boldsymbol{G}(R)$, the set of $R$-rational points of $\boldsymbol{G}$, is identified with the set of $R$-valued matrices $g$ of degree $d$ satisfying

$$
{ }^{t} g J_{W} g=J_{W}, \quad \operatorname{det}(g)=1 .
$$

Let $\mathfrak{g}$ be the Lie algebra of $\boldsymbol{G}$; then $\mathfrak{g}(R)$ is identified with the set of $R$-valued matrices $g$ of degree $d$ satisfying

$$
{ }^{t} g J_{W}+J_{W} g=0 .
$$

2B. Vertices of Bruhat-Tits building of $\boldsymbol{G}$. Let $G=\boldsymbol{G}(k)$ and $\mathfrak{g}=\mathfrak{g}(k)$. Let $\mathscr{B}(G)=\mathscr{B}(\boldsymbol{G}, k)$ be the Bruhat-Tits building of $G$. For $x \in \mathscr{B}(G)$, let $G_{x}$ be the parahoric subgroup attached to $x$ and $G_{x,+}$ the prounipotent radical of $G_{x}$. Let $G_{x}$ be the connected reductive group defined over $\mathfrak{f}$ such that $G_{x} / G_{x,+}$ is the group of $\mathfrak{f}$-rational points of $\mathrm{G}_{x}$. If $F$ is a $G$-facet of $\mathscr{B}_{(}(G)$ and $x \in F$, let $G_{F}=G_{x}$, $G_{F, 0+}=F_{x, 0+}$, and $\mathrm{G}_{F}=\mathrm{G}_{x}$.

Let $\boldsymbol{S}$ be the maximal $k$-split torus of $\boldsymbol{G}$ containing all diagonal matrices in $\boldsymbol{G}, \boldsymbol{B}$ the Borel subgroup of $\boldsymbol{G}$ containing all upper triangular matrices in $\boldsymbol{G}, \boldsymbol{S}=\boldsymbol{S}(k)$, and $B=\boldsymbol{B}(k)$. Let $\Phi$ be the set of roots of $G$ with respect to $S, \Phi^{+}$the set of positive roots of $G$ with respect to $B$, and $\Delta \subset \Phi^{+}$the subset of simple roots of $\Phi^{+}$. Let $\mathfrak{s}$ be the Lie algebra of $\boldsymbol{S}$; then $\mathfrak{s}=\mathfrak{s}(k)$ consists of all diagonal matrices in $\mathfrak{g}$. By taking differentials, roots in $\Phi$ are identified with linear functions on $\mathfrak{s}$.

Identify $\mathfrak{s}$ with $k^{n}$ by the following isomorphism:

$$
s=\operatorname{diag}\left(c_{1}, \ldots, c_{d}\right) \in \mathfrak{s} \mapsto\left(c_{1}, \ldots, c_{n}\right) \in k^{n} ;
$$

here, $n=[d / 2]$. For $i=1, \ldots, n$, the $i$-th coordinate function $e_{i}$ on $k^{n}$ is identified with a linear function on $\mathfrak{s}$, still denoted by $e_{i}$. Let $\gamma, \alpha_{i}(i=1, \ldots, n)$ be positive roots as follows:

$$
\begin{array}{llll} 
& \alpha_{i}=e_{i}-e_{i+1}, & i=1, \ldots, n ; & \\
& \alpha_{n}=e_{n}, & \gamma=e_{1}+e_{2}, & \text { if } \boldsymbol{G}=\mathbf{S O}(2 n+1) ; \\
\text { or } & \alpha_{n}=e_{n-1}+e_{n}, & \gamma=e_{1}+e_{2}, & \text { if } \boldsymbol{G}=\mathbf{S O}(2 n) ; \\
\text { or } & \alpha_{n}=2 e_{n}, & \gamma=2 e_{1}, & \text { if } \boldsymbol{G}=\mathbf{S p}(2 n) .
\end{array}
$$


Then $\Delta=\left\{\alpha_{1}, \ldots, \alpha_{n}\right\}$ and $\gamma$ is the highest root in $\Phi$ with respect $\Delta$.

Let $\Phi_{\text {af }}$ be the set of affine roots of $G$ with respect to $S$. As a subset of affine functions on $\mathfrak{s}$,

$$
\Phi_{\mathrm{af}}=\{\alpha+m \mid \alpha \in \Phi, m \in \mathbb{Z}\} .
$$

Let $\alpha_{0}=1-\gamma \in \Phi_{\mathrm{af}}$ and $\Sigma=\Delta \cup\left\{\alpha_{0}\right\}$. Then every affine root is an integral combination of elements in $\Sigma$.

Let $X^{*}(S)$ be the character group of $S, X_{*}(S)$ the dual group of $X^{*}(S)$, and

$$
\mathfrak{a}:=X_{*}(S) \otimes_{\mathbb{Z}} \mathbb{R} .
$$

Let $A=A(S)$ be the underlying affine space of $\mathfrak{a}$. Then $A$ is an apartment in $\mathscr{B}(G)$. By fixing a hyperspecial point $o \in A$, one can identify $A$ with $\mathfrak{a}$ and elements in $\Phi_{\mathrm{af}}$ with affine functions on $\mathfrak{a}$.

Let $C$ be the fundamental chamber of $A$ defined by

$$
C=\{z \in A \mid 0<\alpha(z)<1 \text { for all } \alpha \in \Sigma\} .
$$

For $\alpha \in \Phi_{\mathrm{af}}$, let $H_{\alpha}=\{z \in A \mid \alpha(z)=0\}$. Then the $H_{\alpha}(\alpha \in \Sigma)$ are walls of $\bar{C}$. For $0 \leq i \leq n$, let $y_{i} \in \bar{C}$, such that $\left\{y_{i}\right\}=\bigcap H_{\alpha_{j}}$. Then the $y_{i}(i=0, \ldots, n)$ are vertices of $\bar{C}$. Let

$$
I_{\mathrm{nsp}}= \begin{cases}\{2, \ldots, n\} & \text { if } \boldsymbol{G}=\mathbf{S O}(2 n+1) \\ \{2, \ldots, n-2\} & \text { if } \boldsymbol{G}=\mathbf{S O}(2 n) \\ \{1, \ldots, n-1\} & \text { if } \boldsymbol{G}=\mathbf{S p}(2 n)\end{cases}
$$

Then $y_{i}$ is not a special vertex (see [Tits 1979]) for all $i \in I_{\mathrm{nsp}}$, and

$$
\mathrm{G}_{y_{i}}(\mathfrak{f}) \simeq \begin{cases}\mathrm{SO}(2 i, \mathfrak{f}) \times \mathrm{SO}(2 n-2 i+1, \mathfrak{f}) & \text { if } \boldsymbol{G}=\mathbf{S O}(2 n+1), \\ \mathrm{SO}(2 i, \mathfrak{f}) \times \mathrm{SO}(2 n-2 i, \mathfrak{f}) & \text { if } \boldsymbol{G}=\mathbf{S O}(2 n), \\ \mathrm{Sp}(2 i, \mathfrak{f}) \times \mathrm{Sp}(2 n-2 i, \mathfrak{f}) & \text { if } \boldsymbol{G}=\mathbf{S p}(2 n) .\end{cases}
$$

2C. On the stable conjugacy classes of maximal tori. If $\boldsymbol{T}$ is a maximal $K$-split $k$-torus of $\boldsymbol{G}$ defined over $k$, then $T=\boldsymbol{T}(k)$ is a maximal unramified torus of $G$ [DeBacker 2006]. In this case, let $\mathscr{B}(T)=\mathscr{B}(\boldsymbol{T}, k)$. By [Adler 1998], choose a $\operatorname{Gal}(K / k)$-equivariant embedding of $\mathscr{B}(\boldsymbol{T}, K)$ into $\mathscr{B}(\boldsymbol{G}, K)$; then $\mathscr{B}(T)$ is identified with a subset of $\mathscr{B}(G)$ :

$$
\mathscr{B}(T)=\mathscr{B}(\boldsymbol{T}, K)^{\Gamma} \subset \mathscr{B}(\boldsymbol{G}, K)^{\Gamma}=\mathscr{B}(G) .
$$

DeBacker [2006] defines a set $I^{m}$ and an equivalence relation “ $~$ " on $I^{m}$, so that there is a one-to-one and onto correspondence between $I^{m} / \sim$ and the set of $G$-conjugacy classes of unramified maximal tori in $G$. Elements in $I^{m}$ are of the form $(F, \mathrm{~T})$, where $F$ is an arbitrary $G$-facet in $\mathscr{B}(G)$ and $\mathrm{T}$ is a maximal 
minisotropic f-torus in $\mathrm{G}_{F}$. Let $C(F, \mathrm{~T})$ be the $G$-conjugacy class of maximal unramified tori in $G$ corresponding to the equivalence class in $I^{m}$ containing $(F, \mathrm{~T})$.

Let $o \in \mathscr{B}(G)$ be one of the special points chosen in Section 2B, to which we associate a conjugacy class of a maximal anisotropic $\mathfrak{f}$-torus in $\mathrm{G}_{o}$ and a conjugacy class in $\boldsymbol{W}\left(\mathrm{G}_{o}\right)$ (see [DeBacker 2006; Carter 1985]). Here $\boldsymbol{W}\left(\mathrm{G}_{o}\right)$ is the Weyl group of $\mathrm{G}_{o}$. Let $\mathrm{T}_{o}$ (resp. $w_{o}$ ) be a representative of the conjugacy class of a maximal anisotropic $\mathfrak{f}$ torus (resp. the $\boldsymbol{W}\left(\mathrm{G}_{o}\right)$-conjugacy class). Then $\left(\{o\}, \mathrm{T}_{o}\right) \in I^{m}$. Take $T=\boldsymbol{T}(k) \in C\left(\{o\}, \mathrm{T}_{o}\right)$; then $T$ is a maximal unramified anisotropic $k$-torus in $G$ (see [DeBacker 2006]).

Let $\mathscr{Y}\left(\mathrm{T}_{o}\right)$ be the subset of $I^{m}$ consisting of elements $(F, \mathrm{~T})$ such that if $\boldsymbol{W}\left(\mathrm{G}_{F}\right)$ is identified with a subgroup of $\boldsymbol{W}\left(\mathrm{G}_{o}\right)$, then $\boldsymbol{W}\left(\mathrm{G}_{F}\right) w_{F} \cap \boldsymbol{W}\left(\mathrm{G}_{o}\right) w_{o} \neq \varnothing$, where $w_{F}$ is a representative of the $W\left(\mathrm{G}_{F}\right)$-conjugacy class corresponding to $\mathrm{T}$. Then $\mathscr{Y}\left(\mathrm{T}_{o}\right)$ depends only on the conjugacy class of $w_{o}$ in $W\left(\mathrm{G}_{o}\right)$. In fact, $\mathscr{Y}\left(\mathrm{T}_{o}\right)$ is the set of $G$-conjugacy classes of maximal unramified anisotropic tori in the stable conjugacy class of $T$ in $G$, which is the stable conjugacy class of maximal unramified tori in $G$ corresponding to $w_{o}$ [ibid., Corollary 4.3.2]. Let " " be the equivalence relation on $\mathscr{S}\left(\mathrm{T}_{o}\right)$ inherited from $I^{m}$

We briefly recall the classification of conjugacy classes in $W\left(G_{o}\right)$. Since $G_{o}$ is split special orthogonal group or symplectic group over $\mathfrak{f}$,

$$
\boldsymbol{W}\left(\mathrm{G}_{o}\right) \simeq \begin{cases}S_{n} \rtimes(\mathbb{Z} / 2 \mathbb{Z})^{n} & \text { if } \mathrm{G}_{o}=\mathrm{SO}(2 n+1) \text { or } \mathrm{Sp}(2 n), \\ S_{n} \rtimes(\mathbb{Z} / 2 \mathbb{Z})^{n-1} & \text { if } \mathrm{G}_{o}=\mathrm{SO}(2 n), n \geq 2 .\end{cases}
$$

Here $S_{n}$ is the $n$-th symmetric group. Conjugacy classes in $\boldsymbol{W}\left(\mathrm{G}_{o}\right)$ are parametrized by the set of pairs of partitions $(\lambda, \mu)$ with $S(\lambda)+S(\mu)=n$; moreover, if $\mathrm{G}_{o}=$ $\mathrm{SO}(2 n), c(\mu)$ is even [Carter 1972, Propositions 24,25$]$. Here, terminology in [Waldspurger 2001] is used: for a partition $\lambda=\left(\lambda_{1}, \ldots, \lambda_{n}, \ldots\right)$,

$$
S(\lambda)=\sum_{i=1}^{\infty} \lambda_{i}, \quad c(\lambda)=\left|\left\{i \geq 1 \mid \lambda_{i} \neq 0\right\}\right| .
$$

In particular, conjugacy classes of anisotropic maximal tori in $\mathrm{G}_{o}(\mathfrak{f})$ are parametrized by the subset consisting of $(\varnothing, \mu)$, with $S(\mu)=n$; if $\mathrm{G}_{o}=\mathrm{SO}(2 n), c(\boldsymbol{\mu})$ is even.

Assume $(\varnothing, \boldsymbol{\mu})$ corresponds to the conjugacy class of $w_{o}$ in $W\left(\mathrm{G}_{o}\right)$, and write

$$
\boldsymbol{\mu}=\left(\mu_{1}, \ldots, \mu_{s}\right), \quad \mu_{1} \geq \cdots \geq \mu_{s} \geq 1,
$$

so that $S(\mu)=n$, and $s$ is even if $G=\operatorname{SO}(2 n)$. Let

$\mathscr{S}(\boldsymbol{\mu})=\left\{\boldsymbol{\mu}^{\prime}=\left(\mu_{j_{1}}, \ldots, \mu_{j_{s-2 m}}\right) \mid\right.$ for some $\left.1 \leq j_{1}<j_{2}<\cdots<j_{s-2 m}, 0 \leq 2 m \leq s\right\}$, if $G=\mathrm{SO}(2 n+1)$ or $\mathrm{SO}(2 n)$;

$\mathscr{S}(\boldsymbol{\mu})=\left\{\boldsymbol{\mu}^{\prime}=\left(\mu_{j_{1}}, \ldots, \mu_{j_{s-m}}\right) \mid\right.$ for some $\left.1 \leq j_{1}<j_{2}<\cdots<j_{s-m}, 0 \leq m \leq s\right\}$, if $G=\operatorname{Sp}(2 n)$; 
For $\boldsymbol{\mu}^{\prime} \in \mathscr{S}(\boldsymbol{\mu})$, define

$$
i:=i_{\boldsymbol{\mu}^{\prime}}:=i\left(\boldsymbol{\mu}^{\prime}\right):=S(\boldsymbol{\mu})-S\left(\boldsymbol{\mu}^{\prime}\right) .
$$

Then $\boldsymbol{W}\left(\mathrm{G}_{o}\right) w_{o} \cap \boldsymbol{W}\left(\mathrm{G}_{y_{i}}\right) \neq \varnothing$. Here $\boldsymbol{W ( \mathrm { G } _ { o } )} w_{o}$ is the conjugacy class of $w_{o}$ and $\boldsymbol{W}\left(\mathrm{G}_{y_{i}}\right)$ is the Weyl group of $\mathrm{G}_{y_{i}}$ identified as a subgroup of $\boldsymbol{W}\left(\mathrm{G}_{o}\right)$. By [DeBacker 2006, Corollary 4.3.2], there is a maximal anisotropic torus $T_{\mu^{\prime}}$ in $G_{y_{i}}(\mathfrak{f})$ that is $\mathrm{G}_{o}(\mathfrak{f})$-conjugate to $\mathrm{T}_{o}$. Hence $\left(\left\{y_{i\left(\boldsymbol{\mu}^{\prime}\right)}\right\}, \mathrm{T}_{\boldsymbol{\mu}^{\prime}}\right) \in \mathscr{Y}\left(\mathrm{T}_{o}\right)$.

Take $T_{\boldsymbol{\mu}^{\prime}} \in C\left(\left\{y_{i\left(\boldsymbol{\mu}^{\prime}\right)}\right\}, \mathrm{T}_{\boldsymbol{\mu}^{\prime}}\right)$; then $T_{\boldsymbol{\mu}^{\prime}}$ is a maximal unramified anisotropic torus in $G$ stably conjugate to $T$ and $\mathscr{B}\left(T_{\boldsymbol{\mu}^{\prime}}\right)=\left\{y_{i_{\boldsymbol{\mu}^{\prime}}}\right\}$. In particular, $\boldsymbol{\mu} \in \mathscr{S}(\boldsymbol{\mu})$. Take $T_{\mu}=T$. Conversely, all $G$-conjugacy classes in the stable conjugacy class of $T$ have a representative of this form.

Lemma 2.1. The set $\left\{\left(\left\{y_{i_{\boldsymbol{\mu}^{\prime}}}\right\}, \mathrm{T}_{\boldsymbol{\mu}^{\prime}}\right) \mid \boldsymbol{\mu}^{\prime} \in \mathscr{S}(\boldsymbol{\mu})\right\}$ is a complete set of representatives of $\mathscr{Y}\left(\mathrm{T}_{o}\right) / \sim$.

Proof. It remains to show that the pairs $\left(\left\{y_{i_{\mu^{\prime}}}\right\}, \mathrm{T}_{\boldsymbol{\mu}^{\prime}}\right)$ are not equivalent to one another, for $\boldsymbol{\mu}^{\prime} \in \mathscr{S}(\boldsymbol{\mu})$. If $i_{\boldsymbol{\mu}^{\prime}}=i_{\boldsymbol{\mu}^{\prime \prime}}$ for distinct $\boldsymbol{\mu}^{\prime}, \boldsymbol{\mu}^{\prime \prime} \in \mathscr{S}(\boldsymbol{\mu})$, then by the choice of $\mathrm{T}_{\boldsymbol{\mu}^{\prime}}$ and $\mathrm{T}_{\boldsymbol{\mu}^{\prime \prime}}, \mathrm{T}_{\boldsymbol{\mu}^{\prime}}$ is not conjugate to $\mathrm{T}_{\boldsymbol{\mu}^{\prime \prime}}$ in $\mathrm{G}_{y_{i_{\boldsymbol{\mu}^{\prime}}}}$; therefore $\left(\left\{y_{i_{\boldsymbol{\mu}^{\prime}}}\right\}, \mathrm{T}_{\boldsymbol{\mu}^{\prime}}\right)$ is not equivalent to $\left(\left\{y_{i_{\boldsymbol{\mu}^{\prime \prime}}}\right\}, \mathrm{T}_{\boldsymbol{\mu}^{\prime \prime}}\right)$.

If $i_{\boldsymbol{\mu}^{\prime}} \neq i_{\boldsymbol{\mu}^{\prime \prime}}$ for $\boldsymbol{\mu}^{\prime}, \boldsymbol{\mu}^{\prime \prime} \in \mathscr{S}(\boldsymbol{\mu})$, we will show $y_{i_{\boldsymbol{\mu}^{\prime}}}$ is not associated to $y_{i_{\boldsymbol{\mu}^{\prime \prime}}}$. As a consequence, $\left(\left\{y_{i_{\boldsymbol{\mu}^{\prime}}}\right\}, \mathrm{T}_{\boldsymbol{\mu}^{\prime}}\right)$ is not equivalent to $\left(\left\{y_{i_{\boldsymbol{\mu}^{\prime \prime}}}\right\}, \mathrm{T}_{\boldsymbol{\mu}^{\prime \prime}}\right)$.

The case for $\boldsymbol{G}=\mathbf{S p}(2 n)$ is trivial, since the vertices $y_{0}, y_{1}, \ldots, y_{n}$ of $\bar{C}$ are not associated to each other.

If $\boldsymbol{G}=\mathbf{S O}(2 n+1)$, among all vertices $y_{0}, y_{1}, \ldots, y_{n}$ of $\bar{C}, y_{0}$ is associated to $y_{1}$, and $y_{0}, y_{2}, \ldots, y_{n}$ are not associated to each other. For $\boldsymbol{\mu}^{\prime} \in \mathscr{Y}(\boldsymbol{\mu})$, if $i_{\boldsymbol{\mu}^{\prime}} \neq 0$, then $i_{\boldsymbol{\mu}^{\prime}} \geq 2$. As a result, $\left(\left\{y_{i_{\boldsymbol{\mu}^{\prime}}}\right\}, \mathrm{T}_{\boldsymbol{\mu}^{\prime}}\right)$ is not equivalent to $\left(\left\{y_{i_{\boldsymbol{\mu}^{\prime \prime}}}\right\}, \mathrm{T}_{\boldsymbol{\mu}^{\prime \prime}}\right)$.

If $\boldsymbol{G}=\mathbf{S O}(2 n)$, among all vertices $y_{0}, y_{1}, \ldots, y_{n}, y_{0}$ is associated to $y_{1}, y_{n-1}$ is associated to $y_{n}$, and $y_{0}, y_{2}, \ldots, y_{n-2}, y_{n}$ are not associated to each other. For $\boldsymbol{\mu}^{\prime} \in \mathscr{S}(\boldsymbol{\mu})$, if $i_{\boldsymbol{\mu}^{\prime}} \neq 0$, then $i_{\boldsymbol{\mu}^{\prime}} \neq 1, i_{\boldsymbol{\mu}^{\prime}} \neq n-1$. Then $\left(\left\{y_{i_{\boldsymbol{\mu}^{\prime}}}\right\}, \mathrm{T}_{\boldsymbol{\mu}^{\prime}}\right)$ is not equivalent to $\left(\left\{y_{i_{\boldsymbol{\mu}^{\prime \prime}}}\right\}, \mathrm{T}_{\boldsymbol{\mu}^{\prime \prime}}\right)$.

2D. L-packet. Keep the notation of the previous subsection. Let $\mathfrak{t}_{\boldsymbol{\mu}}$ (resp. $\left.\mathfrak{t}_{\boldsymbol{\mu}}(K)\right)$ be the Lie algebra of $T_{\boldsymbol{\mu}}\left(\operatorname{resp} . \boldsymbol{T}_{\boldsymbol{\mu}}(K)\right)$. For $s \in \mathbb{Z}$, let $\mathfrak{t}_{\boldsymbol{\mu}, s}\left(\operatorname{resp} . T_{\boldsymbol{\mu}, s}\right)$ be the $s$-th filtration of $\mathfrak{t}_{\boldsymbol{\mu}}$ (resp. $T_{\boldsymbol{\mu}}$ ) [Adler 1998]. Let $r$ be a positive integer, $X_{\boldsymbol{\mu}}$ a good element in $\mathfrak{t}_{\boldsymbol{\mu},-r}$ (i.e., $X_{\boldsymbol{\mu}} \in \mathfrak{t}_{-r}$ ), and for every root $\alpha$ of $\boldsymbol{T}_{\boldsymbol{\mu}}(K)$ in $\boldsymbol{G}(K)$, assume $\mathrm{d} \alpha\left(X_{\boldsymbol{\mu}}\right) \neq 0$. Let $\chi_{\boldsymbol{\mu}}$ be a character of $T_{\boldsymbol{\mu}}$ satisfying $\left.\chi_{\boldsymbol{\mu}}\right|_{T_{\boldsymbol{\mu}, r+1}}=1$,

$$
\chi_{\boldsymbol{\mu}}\left(\exp _{o}(Y)\right)=\psi\left(\operatorname{tr}\left(X_{\boldsymbol{\mu}} Y\right)\right) \quad \text { for all } Y \in \mathfrak{t}_{\boldsymbol{\mu}, r} .
$$

Here $\exp _{o}$ is the mock exponential map defined in [Adler 1998].

Let $\pi_{\chi_{\boldsymbol{\mu}} ; \boldsymbol{\mu}}$ be the supercuspidal representation constructed by using $\chi_{\boldsymbol{\mu}}$ and $X_{\boldsymbol{\mu}}$, $\varphi: \mathscr{W}_{k} \rightarrow{ }^{L} G$ be the $L$-parameter of $\pi_{\chi_{\boldsymbol{\mu}} ; \boldsymbol{\mu}}$ (see [Adler 1998; Reeder 2008]), where 
$W_{k}$ is the Weil group of $k$. For $\boldsymbol{\mu}^{\prime} \in \mathscr{T}(\boldsymbol{\mu})$, let $g \in \boldsymbol{G}(K)_{o}$ be an element such that $T_{\boldsymbol{\mu}^{\prime}}(k)={ }^{g} T_{\boldsymbol{\mu}}(k)$; then $X_{\boldsymbol{\mu}^{\prime}}={ }^{g} X_{\boldsymbol{\mu}}$ is a good element in $\mathfrak{t}_{\boldsymbol{\mu}^{\prime},-r}$. Define a depth $r$ character $\chi_{\boldsymbol{\mu}^{\prime}}$ of $T_{\boldsymbol{\mu}^{\prime}}$ by $\chi_{\boldsymbol{\mu}^{\prime}}:={ }^{g} \chi_{\boldsymbol{\mu}^{\prime}}$; then,

$$
\chi_{\boldsymbol{\mu}^{\prime}}\left(\exp _{y_{i\left(\boldsymbol{\mu}^{\prime}\right)}}(Y)\right)=\psi\left(\operatorname{tr} X_{\boldsymbol{\mu}^{\prime}} Y\right) \text { for all } Y \in \mathfrak{t}_{\boldsymbol{\mu}^{\prime}, r} .
$$

Let $\pi_{\chi_{\boldsymbol{\mu}} ; \boldsymbol{\mu}^{\prime}}$ be the supercuspidal representation of $G$ constructed by using $\chi_{\boldsymbol{\mu}^{\prime}}$ and $X_{\boldsymbol{\mu}^{\prime}}$. Then:

Theorem 2.2 [Reeder 2008]. The set $\Pi^{\prime}(\varphi)=\left\{\pi_{\chi_{\boldsymbol{\mu}} ; \boldsymbol{\mu}^{\prime}} \mid \boldsymbol{\mu}^{\prime} \in \mathscr{S}(\boldsymbol{\mu})\right\}$ is the L-packet associated to $\varphi$.

The main result of this paper concerns nilpotent orbits supporting representations in $\Pi^{\prime}(\varphi)$. Prior to the statement of the main theorems, we recall the classification of $k$-rational nilpotent orbits in $\mathfrak{g}$ [Waldspurger 2001, §I.6] and define a partition $\lambda^{i}$ for every $i \in I_{\mathrm{nsp}}$.

2E. Nilpotent orbits. Let $\lambda=\left(\lambda_{i}\right)_{i \in \mathbb{N}}$ be a sequence of nonnegative integers such that $\lambda_{j}=0$ for $j$ sufficiently large. Define

$S(\lambda)=\sum_{j \geq 1} \lambda_{j}, \quad c(\lambda)=\left|\left\{j \geq 1 \mid \lambda_{j} \neq 0\right\}\right|, \quad c_{i}(\lambda)=\left|\left\{j \mid \lambda_{j}=i\right\}\right|$ for all $i \in \mathbb{N}$.

If $\lambda_{1} \geq \lambda_{2} \geq \cdots, \lambda$ is called a partition. Let $\mathscr{P}$ be the set of all partitions and $\mathscr{P}(n)$ the subset of all $\lambda \in \mathscr{P}$ such that $S(\lambda)=n$. For $\lambda, \boldsymbol{\mu} \in \mathscr{P}$, let $\lambda \cup \boldsymbol{\mu}$ be the unique partition such that $c_{i}(\lambda \cup \boldsymbol{\mu})=c_{i}(\lambda)+c_{i}(\boldsymbol{\mu})$ for all $i \in \mathbb{N}$.

Let $W$ be the vector space defined in Section 2A and $d=\operatorname{dim}_{k} W$. If $\epsilon_{W}=1$, let $\mathscr{P}(W)$ be the set of partitions $\lambda \in \mathscr{P}(d)$ such that $c_{i}$ is even for all even $i$. If $\epsilon_{W}=-1$, let $\mathscr{P}(W)$ be the set of partitions $\lambda \in \mathscr{P}(d)$ so that $c_{i}$ is even for all odd $i$. Let $\operatorname{Nil}_{I}(W)$ be the set of $\left(\lambda,\left(q_{i}\right)\right)$ with $\lambda \in \mathscr{P}(W)$, and let $q_{i}, i \in \mathbb{N}$, be quadratic forms satisfying these conditions:

- If $\epsilon_{W}=1, q_{i}$ is a nondegenerate quadratic form on $k^{c_{i}}$ for $i$ odd, $q_{i}=0$ for $i$ even, moreover the quadratic form $\bigoplus_{i \in \mathbb{N}} q_{i}$ has the same anisotropic kernel as $q_{W}$; here, $q_{W}$ is the quadratic form on $W$ defined by $q_{W}(v)=\langle v, v\rangle$.

- If $\epsilon_{W}=-1, q_{i}$ is a nondegenerate quadratic form on $k^{c_{i}}$ for $i$ even, $q_{i}=0$ for $i$ odd.

Definition 2.3. $\left(\lambda,\left(q_{i}\right)\right) \in \operatorname{Nil}_{I}(W)$ is called exceptional if $\epsilon_{W}=1,4 \mid d$, and $\lambda_{i}$ is even for all $i \in \mathbb{N}$. In this case, $q_{i}=0$ for all $i \in \mathbb{N}$.

Definition 2.4. - If $\epsilon_{W}=-1$, let $\operatorname{Nil}(W)=\operatorname{Nil}_{I}(W)$;

- If $\epsilon_{W}=1,4 \nmid d$, let $\operatorname{Nil}(W)=\operatorname{Nil}_{I}(W)$;

- If $\epsilon_{W}=1,4 \mid d$, let $\operatorname{Nil}(W)$ be the set consisting all nonexceptional $\left(\lambda,\left(q_{i}\right)\right) \in$ $\operatorname{Nil}_{I}(W)$ and $\left(\lambda,\left(q_{i}\right), \varepsilon\right)$ with $\left(\lambda,\left(q_{i}\right)\right)$ exceptional, $\varepsilon= \pm 1$. 
By [Waldspurger 2001], there is a bijective correspondence between $\operatorname{Nil}(W)$ and $\mathfrak{g}_{\text {nil }} / G$, the set of $k$-rational nilpotent orbits. Define a partial order on $\mathscr{P}(n)$ : for $\lambda, \boldsymbol{\mu} \in \mathscr{P}(n), \boldsymbol{\lambda} \geq \boldsymbol{\mu}$ if and only if for all $j \geq 1, \sum_{i=1}^{j} \lambda_{i} \geq \sum_{i=1}^{j} \mu_{i}$.

Definition 2.5. Define a partial order on the set of nilpotent orbits in $\mathfrak{g}: O_{1} \geq O_{2}$ if and only if $\bar{O}_{1} \supset \bar{O}_{2}$. Here the closure is taken with respect to the usual topology in $\mathfrak{g}$.

Lemma 2.6. Let $O_{1}, O_{2}$ be nilpotent orbits in $\mathfrak{g}$ corresponding to $\left(\lambda,\left(q_{i}\right)\right)$ or $(\lambda, \varnothing, \varepsilon)$ and $\left(\mu,\left(q_{i}^{\prime}\right)\right)$ or $\left(\mu, \varnothing, \varepsilon^{\prime}\right)$ respectively. If $O_{1}>O_{2}$, then $\lambda>\boldsymbol{\mu}$.

Proof. The proof is similar to that of Theorem 6.2.5 of [Collingwood and McGovern 1993]. Take arbitrary $X \in O_{1}, Y \in O_{2}$, with $O_{1}, O_{2}$ corresponding to $\left(\lambda,\left(q_{i}\right)\right)$ or $(\lambda, \varnothing, \varepsilon)$ and $\left(\mu,\left(q_{i}^{\prime}\right)\right)$ or $\left(\mu, \varnothing, \varepsilon^{\prime}\right)$ respectively. If $O_{1}>O_{2}$, then $\bar{O}_{1} \supsetneqq \bar{O}_{2}$,

$$
\operatorname{rank}\left(X^{k}\right)>\operatorname{rank}\left(Y^{k}\right) \text { for all } k \geq 1,
$$

since the condition that rank of a matrix be strictly less than a fixed number is a closed condition for the usual topology. Now $\lambda>\boldsymbol{\mu}$ by of [ibid., Lemma 6.2.2],

Example 2.7. Regular nilpotent orbits in $\mathfrak{g}_{\text {nil }}$ are those corresponding to:

- $\left([2 n+1], q_{2 n+1}\right)$, if $\epsilon_{W}=1, d=2 n+1$. Here $q_{2 n+1}$ is the nondegenerate quadratic form on $k$ defined by $q_{2 n+1}(x)=x^{2}$.

- $\left([2 n-1,1],\left(q_{2 n-1}, q_{1}\right)\right)$, if $\epsilon_{W}=1, d=2 n$. Here $q_{2 n-1}, q_{1}$ are nondegenerate quadratic forms on $k$ such that $q_{2 n-1} \oplus q_{1} \simeq q^{\prime}$, where $q^{\prime}$ is the quadratic form on $k^{2}$ defined by $q^{\prime}(x, y)=2 x y$ for all $x, y \in k$.

- ([2n], $\left.q_{2 n}\right)$, if $\epsilon_{W}=-1, d=2 n$. Here $q_{2 n}$ is a nondegenerate quadratic form on $k$.

Let $I_{\mathrm{nsp}}$ be the set defined in (1). For $i \in I_{\mathrm{nsp}}$, let $\lambda^{i}=\boldsymbol{\mu}^{\prime} \cup \boldsymbol{\mu}^{\prime \prime}$ with

$$
\begin{array}{lll}
\boldsymbol{\mu}^{\prime}=[2 i-1,1], & \boldsymbol{\mu}^{\prime \prime}=[2 n-2 i+1], & \text { if } \epsilon_{W}=1, d=2 n+1 ; \\
\boldsymbol{\mu}^{\prime}=[2 i-1,1], & \boldsymbol{\mu}^{\prime \prime}=[2 n-2 i-1,1], & \text { if } \epsilon_{W}=1, d=2 n ; \\
\boldsymbol{\mu}^{\prime}=[2 i], & \boldsymbol{\mu}^{\prime \prime}=[2 n-2 i], & \text { if } \epsilon_{W}=-1, d=2 n .
\end{array}
$$

For $i \notin I_{\mathrm{nsp}}$, let

$$
\lambda^{i}= \begin{cases}{[d]} & \text { if } \epsilon_{W}=1, d=2 n+1, \\ {[d-1,1]} & \text { if } \epsilon_{W}=1, d=2 n, \\ {[d]} & \text { if } \epsilon_{W}=-1, d=2 n .\end{cases}
$$

Lemma 2.8. Let $i \in I_{\mathrm{nsp}}$. Let $O^{\prime}, O^{i}$ be nilpotent orbits in $\mathfrak{g}_{\text {nil }}$ corresponding to $\left(\lambda^{\prime},\left(q_{j}^{\prime}\right)\right)$ or $\left(\lambda^{\prime}, \varnothing, \varepsilon\right)$ and $\left(\lambda^{i},\left(q_{j}\right)\right)$. Assume $O^{\prime}>O^{i}$. Then: 
- If $\boldsymbol{G}=\mathbf{S O}(2 n+1)$, then $\lambda^{\prime}=[2 n+1]$ or $[m, 2 n-m, 1]$ for some odd $m>\max (2 i-1,2 n-2 i+1)$.

- If $\boldsymbol{G}=\mathbf{S O}(2 n)$ and $i \neq n / 2$, then $\boldsymbol{\lambda}^{\prime}=[m, 2 n-m]$ for some odd $m \geq$ $\max (2 i-1,2 n-2 i-1)$, or $\lambda^{\prime}=\left[m, 2 n-m-2,1^{2}\right]$ for some odd $m>$ $\max (2 i-1,2 n-2 i-1)$.

- If $\boldsymbol{G}=\mathbf{S O}(2 n)$ and $i=n / 2$, then $\lambda^{\prime}=\left[n^{2}\right]$, or

$$
\lambda^{\prime}=[m, 2 n-m] \text { or }\left[m, 2 n-m-2,1^{2}\right]
$$

for some odd $m>\max (2 i-1,2 n-2 i-1)$.

- If $\boldsymbol{G}=\mathbf{S p}(2 n)$, then $\boldsymbol{\lambda}^{\prime}=[m, 2 n-m]$ for some even $m>\max (2 i, 2 n-2 i)$.

Proof. Assume $\lambda^{\prime}=\left[\lambda_{1}^{\prime}, \lambda_{2}^{\prime}, \ldots\right] \in \mathscr{P}(W)$, with $\lambda_{1}^{\prime} \geq \lambda_{2}^{\prime} \geq \cdots$. By Lemma 2.6, if $O^{\prime}>O^{i}$, then $\lambda^{\prime}>\lambda^{i}$.

Assume $\boldsymbol{G}=\mathbf{S O}(2 n+1), \lambda^{i}=[2 i-1,1] \cup[2 n-2 i+1]$. First, assume $2 i-1 \geq 2 n-2 i+1, \lambda^{i}=[2 i-1,2 n-2 i+1,1]$.

By definition, $\lambda^{\prime}>\lambda^{i}$ if and only if $\lambda^{\prime} \neq \lambda^{i}$ and

$$
\lambda_{1}^{\prime} \geq 2 i-1, \quad \lambda_{1}^{\prime}+\lambda_{2}^{\prime} \geq 2 n, \quad \lambda_{1}^{\prime}+\lambda_{2}^{\prime}+\lambda_{3}^{\prime}=2 n+1 .
$$

Then $\lambda_{3}^{\prime}=0$ or $\lambda_{3}^{\prime}=1$. If $\lambda_{3}^{\prime}=0, \lambda_{2}^{\prime}=0$, then $\lambda^{\prime}=[2 n+1]>\lambda^{i}$. If $\lambda_{3}^{\prime}=0$, $\lambda_{2}^{\prime} \neq 0$, then $\lambda^{\prime}=\left[\lambda_{1}^{\prime}, 2 n+1-\lambda_{1}^{\prime}\right] \notin \mathscr{P}(W)$, which contradicts the assumption $\lambda^{\prime} \in \mathscr{P}(W)$.

If $\lambda_{3}^{\prime}=1, \lambda^{\prime}=[m, 2 n-m, 1]$ for some $m \geq 2 i-1$. If $m=2 i-1$, then $\lambda^{\prime}=\lambda^{i}$, which contradicts the assumption $\lambda^{\prime} \neq \lambda^{i}$. Hence $m>2 i-1$. If $m$ is even, then $c_{m}\left(\lambda^{\prime}\right)$ is even and $2 n-m=m$; hence $m=n$, and $\lambda^{\prime}=\left[n^{2}, 1\right]$. On the other hand, $\lambda^{\prime}>\lambda^{i}, 2 i-1=2 n-2 i+1=n=m$, which contradicts $m>2 i-1$. In conclusion, $\lambda^{\prime}=[m, 2 n-m, 1]$ for some odd $m>2 i-1$.

Similarly, if $2 n-2 i-1 \geq 2 i-1, \lambda^{\prime}>\lambda^{i}=[2 n-2 i-1,2 i-1,1]$, then $\lambda^{\prime}=[m, 2 n-m, 1]$ for some odd $m>2 n-2 i+1$. This concludes the proof for $\boldsymbol{G}=\mathbf{S O}(2 n+1)$.

Assume $\boldsymbol{G}=\mathbf{S O}(2 n), \lambda^{i}=[2 i-1,1] \cup[2 n-2 i-1,1]$. First, assume $2 i-1>$ $2 n-2 i-1, \lambda^{i}=\left[2 i-1,2 n-2 i-1,1^{2}\right]$.

By definition, $\lambda^{\prime}>\lambda^{i}$ if and only if $\lambda^{\prime} \neq \lambda^{i}$ and $\lambda_{1}^{\prime} \geq 2 i-1, \quad \lambda_{1}^{\prime}+\lambda_{2}^{\prime} \geq 2 n-2, \quad \lambda_{1}^{\prime}+\lambda_{2}^{\prime}+\lambda_{3}^{\prime} \geq 2 n-1, \quad \lambda_{1}^{\prime}+\lambda_{2}^{\prime}+\lambda_{3}^{\prime}+\lambda_{4}^{\prime}=2 n$. Then $\lambda_{4}^{\prime}=0$ or $\lambda_{4}^{\prime}=1$. Assume $\lambda_{4}^{\prime}=0$; then, $\lambda_{3}^{\prime}=0$ or $\lambda_{3}^{\prime}=1$. If $\lambda_{3}^{\prime}=1$, $\lambda_{4}^{\prime}=0$, then $\lambda_{1}^{\prime}$ and $\lambda_{2}^{\prime}$ have different parity, so $\lambda^{\prime} \notin \mathscr{P}(W)$. If $\lambda_{3}^{\prime}=\lambda_{4}^{\prime}=0$, then $\lambda^{\prime}=[m, 2 n-m]$ with $m \geq 2 i-1$. If $m$ is even, then $c_{m}\left(\lambda^{\prime}\right)$ is even, $m=2 n-m=n$. Hence $m=n>2 i-1>2 n-2 i-1$, which has no solution since the second inequality requires $2 i-1>n-1$. In conclusion, if $\lambda_{4}^{\prime}=0$, then $\lambda^{\prime}=[m, 2 n-m]$ for some odd $m \geq 2 i-1$. 
If $\lambda_{4}^{\prime}=1$, then $\lambda_{3}^{\prime}=1, \lambda^{\prime}=\left[m, 2 n-m-2,1^{2}\right]$ for some $m \geq 2 i-1$. If $m=2 i-1$, then $\lambda^{\prime}=\lambda^{i}$ which contradicts the assumption $\lambda^{\prime} \neq \lambda^{i}$. Hence $m>2 i-1$. If $m$ is even, then $c_{m}\left(\lambda^{\prime}\right)$ is even, $m=2 n-m-2=n-1$. Hence $m=n-1>2 i-1>2 n-2 i-1$, which has no solution since the second inequality requires $2 i-1>n-1$. In conclusion, if $\lambda_{4}^{\prime}=1$, then $\lambda^{\prime}=\left[m, 2 n-m-2,1^{2}\right]$ for some odd $m>2 i-1$.

Similarly, if $2 n-2 i-1>2 i-1$, then $\lambda^{\prime}=[m, 2 n-m]$ for some odd $m \geq \max (2 i-1,2 n-2 i-1)$, or $\lambda^{\prime}=\left[m, 2 n-m-2,1^{2}\right]$ for some odd $m>$ $\max (2 i-1,2 n-2 i-1)$.

Assume now $2 i-1=2 n-2 i-1$. Then $n$ is even, $i=n / 2$, and $\lambda^{i}=\left[(n-1)^{2}, 1^{2}\right]$. Assume $\lambda^{\prime}>\lambda^{i}, \lambda \in \mathscr{P}(W)$. Then

$\lambda_{1}^{\prime} \geq n-1, \quad \lambda_{1}^{\prime}+\lambda_{2}^{\prime} \geq 2 n-2, \quad \lambda_{1}^{\prime}+\lambda_{2}^{\prime}+\lambda_{3}^{\prime} \geq 2 n-1, \quad \lambda_{1}^{\prime}+\lambda_{2}^{\prime}+\lambda_{3}^{\prime}+\lambda_{4}^{\prime}=2 n$.

If $\lambda_{1}^{\prime}=n-1$, then $\lambda_{2}^{\prime}=n-1, \lambda^{\prime}=\left[(n-1)^{2}, 1^{2}\right]=\lambda^{i}$, contradicting the assumption $\lambda^{\prime} \neq \lambda^{i}$. Hence $\lambda_{1}^{\prime} \geq n$. If $\lambda_{1}^{\prime}$ is even, then $c_{\lambda_{1}^{\prime}}$ is even, $\lambda_{1}^{\prime}=\lambda_{2}^{\prime}=n$, and $\lambda=\left[n^{2}\right]$. If $m=\lambda_{1}^{\prime}>n$ is odd, then $m>\max (2 i-1,2 n-2 i-1)=n-1$ and $\lambda^{\prime}=[m, 2 n-m]$ or $\left[m, 2 n-m-2,1^{2}\right]$. This concludes the proof for $\boldsymbol{G}=\mathbf{S O}(2 n)$.

Assume $\boldsymbol{G}=\mathbf{S p}(2 n)$. Without loss of generality, assume $2 i \geq 2 n-2 i$; i.e., $i \geq n / 2$. Then $\lambda^{i}=[2 i, 2 n-2 i]$. By definition, $\lambda^{\prime}>\lambda^{i}$ if and only if $\lambda^{\prime} \neq \lambda^{i}$ and

$$
\lambda_{1}^{\prime} \geq 2 i, \quad \lambda_{1}^{\prime}+\lambda_{2}^{\prime}=2 n .
$$

Hence $\lambda=\left[\lambda_{1}^{\prime}, 2 n-\lambda_{1}^{\prime}\right]$. If $\lambda_{1}^{\prime}=2 i$, then $\lambda_{2}^{\prime}=2 n-2 i, \lambda^{\prime}=\lambda^{i}$, which contradicts the assumption $\lambda^{\prime} \neq \lambda^{i}$. Hence $\lambda_{1}^{\prime}>2 i \geq n$. If $\lambda_{1}^{\prime}$ is odd, then $c_{\lambda_{1}^{\prime}} \lambda^{\prime}$ is even, $\lambda_{1}^{\prime}=\lambda_{2}^{\prime}=n$, which contradicts $\lambda_{1}^{\prime}>n$. As a result, $\lambda^{\prime}=[m, 2 n-m]$ with $m=\lambda_{1}^{\prime}>2 i$ even. This concludes the proof for $\boldsymbol{G}=\mathbf{S p}(2 n)$.

2F. Nilpotent support. Let $O^{\prime}$ be a rational nilpotent orbit in $\mathfrak{g} / G$ and fix an element $z \in O^{\prime}$. Let $\left\{z, h, z^{\prime}\right\}$ be an $\mathfrak{s l}_{2}$ triple in $\mathfrak{g}$; i.e., let there be a Lie algebra homomorphism $\phi: \mathfrak{s l}_{2} \rightarrow \mathfrak{g}$ such that

$$
z=\phi\left(\left(\begin{array}{ll}
0 & 0 \\
1 & 0
\end{array}\right)\right), \quad h=\phi\left(\left(\begin{array}{rr}
1 & 0 \\
0 & -1
\end{array}\right)\right), \quad z^{\prime}=\phi\left(\left(\begin{array}{ll}
0 & 1 \\
1 & 0
\end{array}\right)\right) .
$$

For $i \in \mathbb{Z}$, let $\mathfrak{g}_{i}=\{Z \in \mathfrak{g} \mid \operatorname{Ad}(h)(Z)=i Z\}$. Then $z \in \mathfrak{g}_{-2}$ and $\mathfrak{g}=\bigoplus_{i \in \mathbb{Z}} \mathfrak{g}_{i}$.

Define nilpotent subalgebras $\mathfrak{n}_{\geq 1}^{\prime}, \mathfrak{n}_{\geq 2}^{\prime}$ of $\mathfrak{g}$ and unipotent subgroups $N_{\geq 1}^{\prime}, N_{\geq 2}^{\prime}$ of $G$ as follows:

$$
\begin{aligned}
& \mathfrak{n}_{\geq 1}^{\prime}=\bigoplus_{i \geq 1} \mathfrak{g}_{i}, \quad N_{\geq 1}^{\prime}=\exp \left(\mathfrak{n}_{\geq 1}^{\prime}\right), \\
& \mathfrak{n}_{\geq 2}^{\prime}=\bigoplus_{i \geq 2} \mathfrak{g}_{i}, \quad N_{\geq 2}^{\prime}=\exp \left(\mathfrak{n}_{\geq 2}^{\prime}\right) .
\end{aligned}
$$


Let $\psi_{z}$ be the character of $N_{\geq 2}^{\prime}$ defined by

$$
\psi_{z}(Z)=\psi \circ \operatorname{tr}(z \cdot \log Z) \quad\left(Z \in N_{\geq 2}^{\prime}\right) .
$$

Then $\operatorname{Ker}\left(\psi_{z}\right)$ is a subgroup of $N_{\geq 2}^{\prime}$. If $\mathfrak{n}_{\geq 1}^{\prime}=\mathfrak{n}_{\geq 2}^{\prime}$, so $N_{\geq 1}^{\prime}=N_{\geq 2}^{\prime}$, let $S_{z}$ be the character $\psi_{z}$ of $N_{\geq 1}^{\prime}$. If $\mathfrak{n}_{\geq 1}^{\prime} \neq \mathfrak{n}_{\geq 2}^{\prime}$, then $\mathfrak{g}_{1} \neq 0$ and $N_{\geq 1}^{\prime} / \operatorname{Ker}\left(\psi_{z}\right)$ is isomorphic to a Heisenberg group over $\mathfrak{f}$ with center $N_{\geq 2}^{\prime} / \operatorname{Ker}\left(\psi_{z}\right)$. In this case, let $S_{z}$ be the irreducible representation of $N_{\geq 1}^{\prime}$ whose restriction to $N_{\geq 2}^{\prime}$ is a multiple of $\psi_{z}$.

Definition 2.9. Keep the notation above. Following [Mœglin and Waldspurger 1987], denote by $\mathcal{N}_{\text {wh }}(\pi)$ the set of all nilpotent orbits $O^{\prime}$ in $\mathfrak{g} / G$ such that, for some smooth irreducible representation $\pi$ of $G$, we have $\operatorname{Hom}_{N_{\geq 1}^{\prime}}\left(\pi, S_{z}\right) \neq 0$. Let $\mathcal{N}_{\text {wh,max }}(\pi)$ be the subset of maximal elements in $\mathcal{N}_{\text {wh }}(\pi)$ with respect to the inclusion relation of closure of orbits.

\section{Main theorems}

The main results of this paper are the following theorems, whose proofs are given starting on page 185 and page 192, respectively.

Theorem 3.1. Let $\pi \in \Pi^{\prime}(\varphi)$. Assume $\pi=\pi_{\chi_{\boldsymbol{\mu}} ; \boldsymbol{\mu}^{\prime}}$ for some $\boldsymbol{\mu}^{\prime} \in \mathscr{S}(\boldsymbol{\mu}), i=i_{\boldsymbol{\mu}^{\prime}}$. Let $O^{\prime}, O^{i}$ be nilpotent orbits in $\mathfrak{g}$ corresponding to $\left(\lambda^{\prime},\left(q_{j}^{\prime}\right)\right)$ or $\left(\lambda^{\prime}, \phi, \epsilon\right)$ and $\left(\lambda^{i},\left(q_{j}\right)\right)$ respectively, with $O^{\prime}>O^{i}$. Take arbitrary $z \in O^{\prime}$. Then

$$
\operatorname{Hom}_{N_{\geq 1}^{\prime}}\left(\pi, S_{z}\right)=0 .
$$

Theorem 3.2. Let $\pi \in \Pi^{\prime}(\varphi)$. Assume $\pi=\pi_{\chi_{\mu} ; \boldsymbol{\mu}^{\prime}}$ for some $\boldsymbol{\mu}^{\prime} \in \mathscr{S}(\boldsymbol{\mu}), i=$ $i_{\boldsymbol{\mu}^{\prime}}$. Then there is a nilpotent orbit $O^{i}$ corresponding to $\left(\lambda^{i},\left(q_{j}\right)\right)$ such that $O^{i} \in \mathcal{N}_{\text {wh,max }}(\pi)$.

If $i \notin I_{\mathrm{nsp}}$, then $y_{i}$ is special. In this case, Theorem 3.1 is void and Theorem 3.2 is proved in [DeBacker and Reeder 2010].

The subset $\Gamma_{z}$ of $\boldsymbol{\Phi}^{+}$. Assume now $i \in I_{\mathrm{nsp}}$; that is, $\operatorname{rank}(G)$ is large enough for $I_{\text {nsp }}$ to be nonempty. Let $O^{\prime}, O^{i}$ be nilpotent orbits in $\mathfrak{g}$ corresponding to $\left(\lambda^{\prime},\left(q_{j}^{\prime}\right)\right)$ or $\left(\lambda^{\prime}, \phi, \epsilon\right)$ and $\left(\lambda^{i},\left(q_{j}\right)\right)$ respectively, with $O^{\prime}>O^{i}$. In this subsection, we will choose a particular element $z \in O^{\prime}$ such that

$$
N_{\geq 2}^{\prime} \subset B, \quad N_{\geq 4}^{\prime} \subset B .
$$

Here $B$ is the Borel subgroup consisting of upper triangular matrices in $G$ and $N_{\geq j}^{\prime}$ is the object defined in Section $2 \mathrm{~F}$ for any $\mathfrak{s l}_{2}$ triple $\left\{z, h, z^{\prime}\right\}$ attached to $z$ in $\mathfrak{g}$. Let $\Gamma_{z}^{\prime} \subset \Phi^{+}$be the subset of positive roots such that $\alpha \in \Gamma_{z}^{\prime}$ if and only if the root space $\mathfrak{u}_{\alpha} \subset \mathfrak{n}_{\geq 4}^{\prime}$, and let

$$
\Gamma_{z}:=\Phi^{+} \backslash \Gamma_{z}^{\prime}
$$


The following notation is used frequently: let $\boldsymbol{v}=\left(v_{1}, \ldots, v_{s}\right)$ be a sequence of positive integers such that $d=\sum_{j=1}^{s} v_{j}$. Then every matrix $a \in \mathfrak{g l}(d, k)$ can be written in blocks $a=\left(a_{j, \ell}\right)_{j, \ell \leq s}$, with $a_{j j} \in \mathfrak{g l}\left(v_{j}, k\right)$. Let $A_{j}$ be an arbitrary $v_{j+1} \times v_{j}$ matrix for $1 \leq j \leq s-1$, and let $z\left(v ; A_{1}, \ldots, A_{s-1}\right)=\left(z_{j, \ell}\right)_{j, \ell \leq s}$ be the nilpotent element in $\mathfrak{g l}(d, k)$ such that

$$
z_{j, \ell}= \begin{cases}A_{\ell} & j=\ell+1, \\ 0_{v_{j} \times v_{\ell}} & j \neq \ell+1 .\end{cases}
$$

Assume $\boldsymbol{G}=\mathbf{S O}(2 n+1)$. By Lemma $2.8, \boldsymbol{\lambda}^{\prime}=[2 n+1]$ or $[m, 2 n-m, 1]$ with $m$ odd and $m>\max (2 i-1,2 n-2 i+1)$.

First, assume $\lambda^{\prime}=[2 n+1], q_{2 n+1}^{\prime}=q_{2 n+1}$ as in Example 2.7. Let

$$
z=z(\boldsymbol{v} ; 1, \ldots, 1,-1, \ldots,-1),
$$

with $\boldsymbol{v}=\left(1^{2 n+1}\right)$ a regular nilpotent element in $\mathfrak{g}$. Let $\left\{z, h, z^{\prime}\right\}$ be an $\mathfrak{s l}_{2}$ triple attached to $z$ in $\mathfrak{g}$ and $\mathfrak{g}_{j}, \mathfrak{n}_{\geq j}^{\prime}, N_{\geq j}^{\prime}$ the objects defined in Section $2 \mathrm{~F}$. Then, we naturally have

$$
\begin{aligned}
& N_{\geq 2}^{\prime}=\left\{n=\left(n_{j, \ell}\right)_{j, \ell \leq 2 n+1} \in \mathfrak{g} \mid n_{j, \ell}=0_{v_{j} \times v_{\ell}} \text { if } j \geq \ell\right\} \subset B, \\
& N_{\geq 4}^{\prime}=\left\{n=\left(n_{j, \ell}\right)_{j, \ell \leq 2 n+1} \in \mathfrak{g} \mid n_{j, \ell}=0_{v_{j} \times v_{\ell}} \text { if } j \geq \ell-1\right\} \subset B .
\end{aligned}
$$

Let $\Gamma_{z}$ be the subset of $\Phi^{+}$defined in (5); then,

$$
\Gamma_{z}=\left\{\alpha_{j} \mid j=1, \ldots, n\right\} .
$$

Second, assume $m=2 n-1$. Then $\lambda^{\prime}=\left[2 n-1,1^{2}\right], q_{2 n-1}^{\prime}$ is a nondegenerate quadratic form on $k$, identified with a nonzero element in $k^{\times}$, and $q_{1}^{\prime}$ is a nondegenerate quadratic form on $k^{2}$, such that $q_{2 n-1}^{\prime} \oplus q_{1}^{\prime}$ is isometric to the quadratic form on $k^{3}$

$$
(u, v, w) \mapsto 2 u w+v^{2} \quad(u, v, w \in k) .
$$

Let

$$
z=z\left(\boldsymbol{v} ; 1,1, \ldots, 1, A^{*}, A,-1, \ldots,-1\right),
$$

with $v=\left(1^{n-1}, 3,1^{n-1}\right)$,

$$
A^{*}=\left(a_{m}, b_{m}, c_{m}\right)^{t}, \quad A=-\left(c_{m}, b_{m}, a_{m}\right),
$$

such that $A A^{*}=-q_{2 n-1}^{\prime}$. Then $z \in O^{\prime}$, as shown in the Appendix.

Let $\left\{z, h, z^{\prime}\right\}$ be an $\mathfrak{s l}_{2}$ triple attached to $z$ in $\mathfrak{g}$ and $\mathfrak{g}_{j}, \mathfrak{n}_{\geq j}^{\prime}, N_{\geq j}^{\prime}$ the objects defined in Section 2F. Let $s=s(\boldsymbol{v})=2 n-1=m$. It is shown in the Appendix that

$$
\begin{aligned}
& N_{\geq 2}^{\prime}=\left\{n=\left(n_{j, \ell}\right)_{j, \ell \leq s} \in \mathfrak{g} \mid n_{j, \ell}=0_{v_{j} \times v_{\ell}} \text { if } j \geq \ell\right\}, \\
& N_{\geq 4}^{\prime}=\left\{n=\left(n_{j, \ell}\right)_{j, \ell \leq s} \in \mathfrak{g} \mid n_{j, \ell}=0_{v_{j} \times v_{\ell}} \text { if } j \geq \ell-1\right\} ;
\end{aligned}
$$


that is, (4) is satisfied. Let $\Gamma_{z}$ be the subset of $\Phi^{+}$defined in (5); then,

$$
\Gamma_{z}=\left\{\alpha_{j} \mid j=1, \ldots, n-2\right\} \cup\left\{e_{n-1} \pm e_{n}\right\} \cup\left\{e_{n-1}, e_{n}\right\} .
$$

Here the $\alpha_{j}(j=0,1, \ldots n)$ are simple roots defined in Section 2B.

Third, assume $m<2 n-1$. Then $\lambda^{\prime}=[m, 2 n-m, 1]$, and $q_{m}^{\prime}, q_{2 n-m}^{\prime}, q_{1}^{\prime}$ are nondegenerate quadratic forms on $k$ such that $q_{m}^{\prime} \oplus q_{2 n-m}^{\prime} \oplus q_{1}^{\prime}$ is isometric to quadratic form $(u, v, w) \mapsto 2 u w+v^{2}(u, v, w \in k)$. Let

$$
z=z\left(v ; 1 \ldots, 1, a^{*}, 1_{2}, \ldots, 1_{2}, A^{*}, A,-1_{2}, \ldots,-1_{2}, a,-1, \ldots,-1\right),
$$

with $v=\left(1^{m-n}, 2^{n-(m+1) / 2}, 3,2^{n-(m+1) / 2}, 1^{m-n}\right), a^{*}=(1,0)^{t}, a=-(0,1)$,

$$
A^{*}=\left(\begin{array}{ll}
a_{m} & a_{2 n-m} \\
b_{m} & b_{2 n-m} \\
c_{m} & c_{2 n-m}
\end{array}\right), \quad A=-\left(\begin{array}{ccc}
c_{2 n-m} & b_{2 n-m} & a_{2 n-m} \\
c_{m} & b_{m} & a_{m}
\end{array}\right),
$$

such that

$$
A A^{*}=-\left(\begin{array}{cc}
0 & q_{2 n-m}^{\prime} \\
q_{m}^{\prime} & 0
\end{array}\right)
$$

Working as in the Appendix, given $z \in O^{\prime}$, let $\left\{z, h, z^{\prime}\right\}$ be an $\mathfrak{s l}_{2}$ triple attached to $z$ in $\mathfrak{g}$ and let $\mathfrak{g}_{j}, \mathfrak{n}_{\geq j}^{\prime}, N_{\geq j}^{\prime}$ be the objects defined in Section $2 \mathrm{~F}$. Let $s=s(v)=m$; then, (4) is satisfied:

$$
\begin{aligned}
& N_{\geq 2}^{\prime}=\left\{n=\left(n_{j, \ell}\right)_{j, \ell \leq s} \in \mathfrak{g} \mid n_{j, \ell}=0_{v_{j} \times v_{\ell}} \text { if } j \geq \ell\right\} \subset B, \\
& N_{\geq 4}^{\prime}=\left\{n=\left(n_{j, \ell}\right)_{j, \ell \leq s} \in \mathfrak{g} \mid n_{j, \ell}=0_{v_{j} \times v_{\ell}} \text { if } j \geq \ell-1\right\} \subset B .
\end{aligned}
$$

Let $\Gamma_{z} \subset \Phi^{+}$be the subset of positive roots defined in (5); then,

$$
\begin{aligned}
\Gamma_{z}= & \left\{\alpha_{j} \mid j=1, \ldots, m-n\right\} \cup\left\{e_{m-n}-e_{m-n+2}\right\} \\
& \cup\left\{\alpha_{m-n+2 j-1} \mid j=1, \ldots, n-(m+1) / 2\right\} \\
& \cup \bigcup_{j=1}^{n-\frac{m+3}{2}}\left\{e_{m-n+2 j-1}-e_{m-n+2 j+1}, e_{m-n+2 j-1}-e_{m-n+2 j+2}\right\} \\
& \cup \bigcup_{j=1}^{n-\frac{m+3}{2}}\left\{e_{m-n+2 j}-e_{m-n+2 j+1}, e_{m-n+2 j}-e_{m-n+2 j+2}\right\} \\
& \cup\left\{e_{n-2} \pm e_{n}\right\} \cup\left\{e_{n-1} \pm e_{n}\right\} \cup\left\{e_{n-2}, e_{n-1}, e_{n}\right\} .
\end{aligned}
$$

Assume $\boldsymbol{G}=\mathbf{S O}(2 n)$. By Lemma $2.8, \boldsymbol{\lambda}^{\prime}$ is one of $\left[n^{2}\right],[m, 2 n-m]$, or $\left[m, 2 n-m-2,1^{2}\right]$ for some odd $m \geq \max (2 i-1,2 n-2 i-1)$.

First, assume $m=2 n-3$ and $\lambda^{\prime}=\left[m, 2 n-m-2,1^{2}\right]=\left[2 n-3,1^{3}\right]$. Then $q_{2 n-3}^{\prime}$ and $q_{1}^{\prime}$ are nondegenerate quadratic forms on $k$ and $k^{3}$, respectively, such 
that $q_{2 n-3}^{\prime} \oplus q_{1}^{\prime}$ is isometric to the quadratic form on $k^{4}$ defined by $(u, v, w, x)=$ $2 u x+2 v w(u, v, w, x \in k)$. Let $\boldsymbol{v}=\left(1^{n-2}, 4,1^{n-2}\right), s=s(\boldsymbol{v})=2 n-3=m$, and $z=z\left(v ; 1, \ldots, 1, A^{*}, A,-1, \ldots,-1\right)$, with

$$
A^{*}=\left(a_{2 n-3}, b_{2 n-3}, c_{2 n-3}, d_{2 n-3}\right)^{t}, \quad A=-\left(d_{2 n-3}, c_{2 n-3}, b_{2 n-3}, a_{2 n-3}\right)
$$

satisfying $A A^{*}=-q_{2 n-3}^{\prime}$. Similar to that in the Appendix, $z \in O^{\prime}$. Let $\left\{z, h, z^{\prime}\right\}$ be an $\mathfrak{s l}_{2}$ triple attached to $z$ in $\mathfrak{g}$ and $\mathfrak{g}_{j}, \mathfrak{n}_{\geq j}^{\prime}, N_{\geq j}^{\prime}$ the objects defined in Section $2 \mathrm{~F}$. Then

$$
\begin{aligned}
& N_{\geq 2}^{\prime}=\left\{n=\left(n_{j, \ell}\right)_{j, \ell \leq s} \in \mathfrak{g} \mid n_{j, \ell}=0_{v_{j} \times v_{\ell}} \text { if } j \geq \ell\right\} \subset B, \\
& N_{\geq 4}^{\prime}=\left\{n=\left(n_{j, \ell}\right)_{j, \ell \leq s} \in \mathfrak{g} \mid n_{j, \ell}=0_{v_{j} \times v_{\ell}} \text { if } j \geq \ell-1\right\} \subset B .
\end{aligned}
$$

Let $\Gamma_{z} \subset \Phi^{+}$be the subset of positive roots defined in (5); then,

$$
\Gamma_{z}=\left\{\alpha_{j} \mid j=1, \ldots, n-3\right\} \cup\left\{e_{n-2} \pm e_{n-1}\right\} \cup\left\{e_{n-2} \pm e_{n}\right\} \cup\left\{e_{n-1} \pm e_{n}\right\}
$$

Second, assume $\lambda^{\prime}=\left[m, 2 n-m-2,1^{2}\right]$ for some odd $\left.m<2 n-3, m\right\rangle$ $\max (2 i-1,2 n-2 i-1)$. Since $m>2 n-m-2>1, q_{m}^{\prime}, q_{2 n-m-2}^{\prime}$ are quadratic forms on $k$ and $q_{1}^{\prime}$ is a quadratic form on $k^{2}$ such that $q_{m}^{\prime} \oplus q_{2 n-m-2}^{\prime} \oplus q_{1}^{\prime}$ is isometric to the quadratic form on $k^{4}$ defined by

$$
(u, v, w, x)=2 u x+2 v w \quad(u, v, w, x \in k) .
$$

Let $\boldsymbol{v}=\left(1^{m-n+1}, 2^{n-\frac{m+3}{2}}, 4,2^{n-\frac{m+3}{2}}, 1^{m-n+1}\right), s=s(v)=m$, and

$$
z=z\left(\boldsymbol{v} ; 1, \ldots, 1, a^{*}, 1_{2}, \ldots, 1_{2}, A^{*}, A,-1_{2}, \ldots,-1_{2}, a,-1, \ldots,-1\right),
$$

with $a^{*}=(1,0)^{t}, a=-(0,1)$,

$$
A^{*}=\left(\begin{array}{ll}
a_{m} & a_{2 n-m-2} \\
b_{m} & b_{2 n-m-2} \\
c_{m} & c_{2 n-m-2} \\
d_{m} & c_{2 n-m-2}
\end{array}\right), A=-\left(\begin{array}{cccc}
d_{2 n-m-2} & c_{2 n-m-2} & b_{2 n-m-2} & a_{2 n-m-2} \\
d_{m} & c_{m} & b_{m} & a_{m}
\end{array}\right),
$$

such that

$$
A A^{*}=-\left(\begin{array}{cc}
0 & q_{2 n-m-2}^{\prime} \\
q_{m}^{\prime} & 0
\end{array}\right)
$$

Working as in the Appendix, given $z \in O^{\prime}$, let $\left\{z, h, z^{\prime}\right\}$ be an $\mathfrak{s l}_{2}$ triple attached to $z$ in $\mathfrak{g}$ and let $\mathfrak{g}_{j}, \mathfrak{n}_{\geq j}^{\prime}, N_{\geq j}^{\prime}$ be the objects defined in Section $2 \mathrm{~F}$. Then

$$
\begin{aligned}
& N_{\geq 2}^{\prime}=\left\{n=\left(n_{j, \ell}\right)_{j, \ell \leq s} \in \mathfrak{g} \mid n_{j, \ell}=0_{v_{j} \times v_{\ell}} \text { if } j \geq \ell\right\} \subset B, \\
& N_{\geq 4}^{\prime}=\left\{n=\left(n_{j, \ell}\right)_{j, \ell \leq s} \in \mathfrak{g} \mid n_{j, \ell}=0_{v_{j} \times v_{\ell}} \text { if } j \geq \ell-1\right\} \subset B .
\end{aligned}
$$

Let $\Gamma_{z} \subset \Phi^{+}$be the subset of positive roots defined in (5); then, 
(13)

$$
\begin{aligned}
\Gamma_{z}= & \left\{\alpha_{j} \mid j=1, \ldots, m-n+1\right\} \cup\left\{e_{m-n+1}-e_{m-n+3}\right\} \\
& \cup\left\{\alpha_{m-n+1+2 j-1} \mid j=1, \ldots, n-(m+3) / 2\right\} \\
& \cup \bigcup_{j=1}^{n-\frac{m+5}{2}}\left\{e_{m-n+1+2 j-1}-e_{m-n+1+2 j+1}, e_{m-n+1+2 j-1}-e_{m-n+1+2 j+2}\right\} \\
& \cup \bigcup_{j=1}^{n-\frac{m+5}{2}}\left\{e_{m-n+1+2 j}-e_{m-n+1+2 j+1}, e_{m-n+1+2 j}-e_{m-n+1+2 j+2}\right\} \\
& \cup\left\{e_{n-3} \pm e_{n-1}, e_{n-3} \pm e_{n}\right\} \cup\left\{e_{n-2} \pm e_{n-1}, e_{n-2} \pm e_{n}\right\} \\
& \cup\left\{e_{n-1} \pm e_{n}\right\} .
\end{aligned}
$$

Third, assume $\lambda^{\prime}=[m, 2 n-m]$ for some odd $m \geq n$. If $m>n$, then $q_{m}^{\prime}, q_{2 n-m}^{\prime}$ are quadratic forms on $k$ such that $q_{m}^{\prime} \oplus q_{2 n-m}^{\prime}$ is isometric to the quadratic form on $k^{2}$ defined by $(u, w) \mapsto 2 u w$. If $m=n$ is odd, then $\lambda^{\prime}=\left[n^{2}\right]$, and $q_{n}^{\prime}$ is the quadratic form on $k^{2}$ isometric to the quadratic form on $k^{2}$ defined by $(u, w) \mapsto 2 u w$.

Let $\boldsymbol{v}=\left(1^{m-n}, 2^{2 n-m}, 1^{m-n}\right), s=s(\boldsymbol{v})=m$, and

$z= \begin{cases}z\left(\boldsymbol{v} ; 1_{2}, \ldots, 1_{2}, A^{*}, A,-1_{2}, \ldots,-1_{2}\right), & m=n, \\ z\left(v ; 1, \ldots, 1, a^{*}, 1_{2}, \ldots, 1_{2}, A^{*}, A,-1_{2}, \ldots,-1_{2}, a,-1, \ldots,-1\right), & m>n,\end{cases}$

with $a^{*}=(1,0)^{t}, a=-(0,1)$,

$$
A^{*}=\left(\begin{array}{ll}
a_{m} & a_{2 n-m} \\
b_{m} & b_{2 n-m}
\end{array}\right), \quad A=-\left(\begin{array}{cc}
b_{2 n-m} & a_{2 n-m} \\
b_{m} & a_{m}
\end{array}\right),
$$

satisfying

$$
A A^{*}=-\left\{\begin{array}{cc}
\left(\begin{array}{cc}
0 & q_{2 n-m}^{\prime} \\
q_{m}^{\prime} & 0
\end{array}\right) & \text { if } m>n, \\
-\left(\begin{array}{ll}
2 & 0 \\
0 & 2
\end{array}\right) & \text { if } m=n
\end{array}\right.
$$

Working as in the Appendix, given $z \in O^{\prime}$, let $\left\{z, h, z^{\prime}\right\}$ be an $\mathfrak{s l}_{2}$ triple attached to $z$ in $\mathfrak{g}$ and let $\mathfrak{g}_{j}, \mathfrak{n}_{\geq j}^{\prime}, N_{\geq j}^{\prime}$ be the objects defined in Section $2 \mathrm{~F}$. Then

$$
\begin{aligned}
& N_{\geq 2}^{\prime}=\left\{n=\left(n_{j, \ell}\right)_{j, \ell \leq s} \in \mathfrak{g} \mid n_{j, \ell}=0_{v_{j} \times v_{\ell}} \text { if } j \geq \ell\right\} \subset B, \\
& N_{\geq 4}^{\prime}=\left\{n=\left(n_{j, \ell}\right)_{j, \ell \leq s} \in \mathfrak{g} \mid n_{j, \ell}=0_{v_{j} \times v_{\ell}} \text { if } j \geq \ell-1\right\} \subset B .
\end{aligned}
$$

Let $\Gamma_{z} \subset \Phi^{+}$be the subset of positive roots defined in (5); then, 
(14)

$$
\begin{aligned}
\Gamma_{z}= & \left\{\alpha_{j} \mid j=1, \ldots, m-n\right\} \cup\left\{e_{m-n}-e_{m-n+2}\right\} \\
& \cup\left\{\alpha_{m-n+2 j-1} \mid j=1, \ldots, n-(m+1) / 2\right\} \\
& \cup \bigcup_{j=1}^{n-\frac{m+3}{2}}\left\{e_{m-n+2 j-1}-e_{m-n+2 j+1}, e_{m-n+2 j-1}-e_{m-n+2 j+2}\right\} \\
& \cup \bigcup_{j=1}^{n-\frac{m+3}{2}}\left\{e_{m-n+2 j}-e_{m-n+2 j+1}, e_{m-n+1+2 j}-e_{m-n+2 j+2}\right\} \\
& \cup\left\{e_{n-1} \pm e_{n}\right\} \cup\left\{e_{n-2} \pm e_{n}\right\} .
\end{aligned}
$$

Fourth, assume $n$ is even and $\lambda^{\prime}=\left[n^{2}\right]$. Let $\boldsymbol{v}=\left(2^{n}\right)$,

$$
z=z\left(v ; 1_{2}, \ldots, 1_{2}, A,-1_{2}, \ldots,-1_{2}\right),
$$

with $A=\operatorname{diag}(1,-1)$. Working as in the Appendix, take $z_{\varepsilon} \in O_{\varepsilon}^{\prime}$, where $O_{\varepsilon}^{\prime}$ is the nilpotent orbit corresponding to $\left(\lambda^{\prime}, \varnothing, \varepsilon\right)$ for some $\varepsilon=1$ or -1 . Let $\left\{z_{\varepsilon}, h_{\varepsilon}, z_{\varepsilon}\right\}$ be an $\mathfrak{s l}_{2}$ triple attached to $z_{\varepsilon}$ in $\mathfrak{g}$, and $\mathfrak{g}_{j}, \mathfrak{n}_{\geq j}^{\prime}, N_{\geq j}^{\prime}$ the objects defined in Section $2 \mathrm{~F}$. Then

$$
\begin{aligned}
& N_{\geq 2}^{\prime}=\left\{u=\left(u_{j, \ell}\right)_{j, \ell \leq n} \in \mathfrak{g} \mid u_{j, \ell}=0_{v_{j} \times v_{\ell}} \text { if } j \geq \ell\right\} \subset B, \\
& N_{\geq 4}^{\prime}=\left\{u=\left(u_{j, \ell}\right)_{j, \ell \leq n} \in \mathfrak{g} \mid u_{j, \ell}=0_{v_{j} \times v_{\ell}} \text { if } j \geq \ell-1\right\} \subset B .
\end{aligned}
$$

Let $\Gamma_{z_{\varepsilon}} \subset \Phi^{+}$be the subset of positive roots defined in (5) for $z_{\varepsilon}$; then,

$$
\begin{aligned}
\Gamma_{z_{\epsilon}}= & \left\{\alpha_{2 j-1} \mid j=1, \ldots, n / 2-1\right\} \cup\left\{e_{n-1} \pm e_{n}\right\} \\
& \cup \bigcup_{j=1}^{\frac{n}{2}-1}\left\{e_{2 j-1}-e_{2 j+1}, e_{2 j-1}-e_{2 j+2}, e_{2 j}-e_{2 j+1}, e_{2 j}-e_{2 j+2}\right\} .
\end{aligned}
$$

Let $w_{0}=\left(a_{\ell, \ell^{\prime}}\right)_{2 n \times 2 n}$ be the element in $\mathbf{O}(2 n)$ satisfying

$$
\begin{cases}a_{n, n+1}=a_{n+1, n}=a_{j, j}=1 & \text { if } 1 \leq j \leq 2 n, j \neq n, j \neq n+1 \\ a_{\ell, \ell^{\prime}}=0 & \text { otherwise. }\end{cases}
$$

Let $z_{-\varepsilon}=w_{0} z_{\varepsilon} w_{0}^{-1}$; then $z_{-\varepsilon} \in O_{-\epsilon}^{\prime}$, where $O_{-\epsilon}^{\prime}$ is the nilpotent orbit corresponding to $\left(\lambda^{\prime}, \phi,-\varepsilon\right)$. Let $\left\{z_{-\varepsilon}, h_{-\varepsilon}, z_{-\varepsilon}\right\}$ be an $\mathfrak{s l}_{2}$ triple attached to $z_{-\varepsilon}$ in $\mathfrak{g}$ and $\mathfrak{g}_{j}^{\prime \prime}$, $\mathfrak{n}_{\geq j}^{\prime \prime}, N_{\geq j}^{\prime \prime}$ the objects defined in Section $2 \mathrm{~F}$. Then

$$
N_{\geq 2}^{\prime \prime}=w_{0} N_{\geq 2}^{\prime} w_{0}^{-1} \subset B, \quad N_{\geq 4}^{\prime \prime}=w_{0} N_{\geq 4}^{\prime} w_{0}^{-1} \subset B .
$$

Let $\Gamma_{z_{-\varepsilon}} \subset \Phi^{+}$be the subset of positive roots defined in (5) for $z_{-\varepsilon}$, then

$$
\Gamma_{z_{-\varepsilon}}=\left\{e_{n-3}+e_{n}, e_{n-2}+e_{n}\right\} \cup \Gamma_{z_{\varepsilon}} \backslash\left\{e_{n-3}-e_{n}, e_{n-2}-e_{n}\right\} .
$$


Assume $\boldsymbol{G}=\mathbf{S p}(2 n)$. By Lemma 2.8, $\boldsymbol{\lambda}^{\prime}=[m, 2 n-m]$ for some even $m>$ $\max (2 i, 2 n-2 i)$. Then $m>2 n-m$, and $q_{m}^{\prime}, q_{2 n-m}^{\prime}$ are nondegenerate quadratic forms on $k$. Let $\boldsymbol{v}=\left(1^{m-n}, 2^{2 n-m}, 1^{m-n}\right), s=s(\boldsymbol{v})=m$, and

$$
z=z\left(\boldsymbol{v} ; 1, \ldots, 1, a^{*}, 1_{2}, \ldots, 1_{2}, A,-1_{2}, \ldots,-1_{2}, a,-1, \ldots,-1\right),
$$

with $a^{*}=(1,0)^{t}, a=-(0,1), A=\left(\begin{array}{ll}b & a \\ a & c\end{array}\right)$, such that $q_{m}^{\prime} \oplus q_{2 n-m}^{\prime}$ is isometric to the quadratic form given by the symmetric matrix $A$.

Working as in the Appendix, given $z \in O^{\prime}$, let $\left\{z, h, z^{\prime}\right\}$ be an $\mathfrak{s l}_{2}$ triple attached to $z$ in $\mathfrak{g}$ and let $\mathfrak{g}_{j}, \mathfrak{n}_{\geq j}^{\prime}, N_{\geq j}^{\prime}$ be the objects defined in Section $2 \mathrm{~F}$. Then

$$
\begin{aligned}
& N_{\geq 2}^{\prime}=\left\{u=\left(u_{j, \ell}\right)_{j, \ell \leq s} \in \mathfrak{g} \mid u_{j, \ell}=0_{v_{j} \times v_{\ell}} \text { if } j \geq \ell\right\} \subset B, \\
& N_{\geq 4}^{\prime}=\left\{u=\left(u_{j, \ell}\right)_{j, \ell \leq s} \in \mathfrak{g} \mid u_{j, \ell}=0_{v_{j} \times v_{\ell}} \text { if } j \geq \ell-1\right\} \subset B .
\end{aligned}
$$

Let $\Gamma_{z} \subset \Phi^{+}$be the subset of positive roots defined in (5) for $z$; then,

$$
\begin{aligned}
\Gamma_{z}= & \left\{\alpha_{j} \mid j=1, \ldots, m-n\right\} \cup\left\{e_{m-n}-e_{m-n+2}\right\} \\
& \cup\left\{\alpha_{m-n+2 j-1} \mid j=1, \ldots, n-(m) / 2\right\} \\
& \cup \bigcup_{j=1}^{n-\frac{m}{2}-1}\left\{e_{m-n+2 j-1}-e_{m-n+2 j+1}, e_{m-n+2 j-1}-e_{m-n+2 j+2}\right\} \\
& \cup \bigcup_{j=1}^{n-\frac{m}{2}-1}\left\{e_{m-n+2 j}-e_{m-n+2 j+1}, e_{m-n+1+2 j}-e_{m-n+2 j+2}\right\} \\
& \cup\left\{e_{n-1}+e_{n}, 2 e_{n-1}, 2 e_{n}\right\} .
\end{aligned}
$$

Proof of Theorem 3.1. We keep the notation used so far in this section and in Section 2B. For $i \in I_{\mathrm{nsp}}$, let

$$
\Sigma_{i}=\left\{\alpha_{j} \mid j=1, \ldots, n, j \neq i\right\} \cup\{-\gamma\},
$$

which is a set of simple roots of a root subsystem of $\Phi$. Let $O^{\prime}, O^{i}$ be nilpotent orbits in $\mathfrak{g}$ corresponding to $\left(\lambda^{\prime},\left(q_{j}^{\prime}\right)\right)$ or $\left(\lambda^{\prime}, \phi, \epsilon\right)$ and $\left(\lambda^{i},\left(q_{j}\right)\right)$ respectively, with $O^{\prime}>O^{i}$. Let $z \in O^{\prime}, \Gamma_{z} \subset \Phi^{+}$be as defined (6), (8), (10), (15), and set $\Gamma_{z}^{\prime}=\Phi^{+} \backslash \Gamma_{z}$.

Lemma 3.3. Let $w$ be a Weyl element of $G$ such that $w^{-1}\left(\Sigma_{i}\right) \subset \Phi^{+}$. Then $w^{-1}\left(\Sigma_{i}\right) \cap \Gamma_{z}^{\prime} \neq \varnothing$.

Proof. First assume $\boldsymbol{G}=\mathbf{S O}(2 n+1)$. Then $-\gamma=-e_{1}-e_{2}, \alpha_{j}=e_{j}-e_{j+1}$ for $j=$ $1, \ldots, n-1$, and $\alpha_{n}=e_{n}$. Let $w$ be a Weyl element of $G$ such that $w^{-1}\left(\Sigma_{i}\right) \subset \Phi^{+}$; then, there is a permutation $\sigma$ of $\{1,2, \ldots, n\}$ satisfying $\sigma(1)>\sigma(2)>\cdots>\sigma(i)$, 
$\sigma(i+1)<\sigma(i+2)<\cdots<\sigma(n)$, such that

$$
w^{-1}\left(e_{j}\right)=\left\{\begin{aligned}
\pm e_{\sigma(1)} & \text { if } j=1 \\
-e_{\sigma(j)} & \text { if } 2 \leq j \leq i, \\
e_{\sigma(j)} & \text { if } i+1 \leq j \leq n .
\end{aligned}\right.
$$

Assume on the contrary that $w^{-1}\left(\Sigma_{i}\right) \cap \Gamma_{z}^{\prime}=\varnothing$; then

$$
w^{-1}\left(\Sigma_{i}\right) \subset \Gamma_{z} .
$$

If $i=n$, then $\lambda^{i}=\left[2 n-1,1^{2}\right], \Sigma_{n}=\left\{\alpha_{j} \mid 1 \leq j<n\right\} \cup\{-\gamma\}$. Then by Lemma 2.8, $\lambda^{\prime}=[2 n+1]$ and $q_{2 n+1}^{\prime}=q_{2 n+1}$, and by (7), $\Gamma_{z}=\left\{\alpha_{j} \mid j=1, \ldots, n\right\}$. If $w$ satisfies (19) and (20), then $\sigma(j)=n+1-j$,

$$
w^{-1}\left(e_{1}\right)= \pm e_{n}, \quad w^{-1}\left(e_{j}\right)=-e_{n+1-j} \quad(1<j \leq n) .
$$

As a result, $w^{-1}\left(\Sigma_{n}\right)=\left\{\alpha_{j} \mid 1 \leq j<n\right\} \cup\left\{e_{n-1}+e_{n}\right\} \not \subset \Gamma_{z}$, which contradicts (20). Hence $w^{-1}\left(\Sigma_{n}\right) \cap \Gamma_{z}^{\prime} \neq \varnothing$.

If $i<n$, by Lemma $2.8, \lambda^{\prime}=[2 n+1]$ or $[m, 2 n-m, 1]$ for some odd $m>$ $\max (2 i-1,2 n-2 i+1)$. Let $w$ be a Weyl element satisfying (19) and (20). Since $\pm e_{1}-e_{2}, e_{n} \in \Sigma_{i}$, we have

$$
w^{-1}\left( \pm e_{1}-e_{2}\right)=e_{\sigma(2)} \pm e_{\sigma(1)} \in \Gamma_{z}, \quad w^{-1}\left(e_{n}\right)=e_{\sigma(n)} \in \Gamma_{z} .
$$

If $\lambda^{\prime}=[2 n+1]$, then $\Gamma_{z}=\left\{\alpha_{j} \mid 1 \leq j \leq n\right\}$ and $e_{\sigma(2)}+e_{\sigma(1)} \notin \Gamma_{z}$, which contradicts (21).

If $\lambda^{\prime}=[m, 2 n-m, 1], m=2 n-1$, then $\Gamma_{z}$ is the set in (9). By (21), $\sigma(2)=n-1$, $\sigma(1)=n$, while $\sigma(n)=n$ or $n-1$, which is impossible since $\sigma$ is a permutation.

If $\lambda^{\prime}=[m, 2 n-m, 1], m<2 n-1$, then $\Gamma_{z}$ is the set in (11). By (21), $\sigma(1)=n$, $\{\sigma(2), \sigma(n)\}=\{n-2, n-1\}$. If $e_{2}-e_{3}, e_{n-1}-e_{n} \in \Sigma_{i}$, then by (20),

$$
w^{-1}\left(e_{2}-e_{3}\right)=e_{\sigma(3)}-e_{\sigma(2)} \in \Gamma_{z}, \quad w^{-1}\left(e_{n-1}-e_{n}\right)=e_{\sigma(n-1)}-e_{\sigma(n)} \in \Gamma_{z} .
$$

Then $\{\sigma(3), \sigma(n-1)\}=\{n-4, n-3\}$. Since $m>\max (2 i-1,2 n-2 i+1)$, we have

$$
n-\frac{m+1}{2}<\min (n-i, i-1),
$$

so the procedure can be repeated $n-\frac{m+1}{2}$ times. Then, for $\ell=2, \ldots, n-\frac{m-1}{2}$,

$$
\{\sigma(\ell), \sigma(n+2-\ell)\}=\{n-2(\ell-1), n-2(\ell-1)+1\} .
$$

In particular, for $\ell_{0}=n-\frac{m-1}{2}$ and $n+2-\ell_{0}=\frac{m+3}{2}$,

$$
\left\{\sigma\left(\ell_{0}\right), \sigma\left(n+2-\ell_{0}\right)\right\}=\left\{\sigma\left(\ell_{0}\right), \sigma\left(\frac{m+3}{2}\right)\right\}=\{m-n+1, m-n+2\} .
$$


Since $m>2 i-1$, we have $m>2 n-2 i+1$,

$\ell_{0}=n-\frac{m-1}{2}<i, \quad i+1<\frac{m+3}{2}=n+2-\ell_{0}, \quad e_{\ell_{0}}-e_{\ell_{0}+1}, e_{\frac{m+1}{2}}-e_{\frac{m+3}{2}} \in \Sigma_{i}$.

By (20),

$$
\begin{gathered}
w^{-1}\left(e_{\ell_{0}}-e_{\ell_{0}+1}\right)=e_{\sigma\left(\ell_{0}+1\right)}-e_{\sigma\left(\ell_{0}\right)} \in \Gamma_{z}, \\
w^{-1}\left(e_{\frac{m+1}{2}}-e_{\frac{m+3}{2}}\right)=e_{\sigma\left(\frac{m+1}{2}\right)}-e_{\sigma\left(\frac{m+3}{2}\right)} \in \Gamma_{z} .
\end{gathered}
$$

Then $\sigma\left(\ell_{0}+1\right)=\sigma\left(\frac{1}{2}(m+1)\right)=m-n$, which contradicts the assumption that $\sigma$ is a permutation, for $\ell_{0}+1 \leq i,(m+1) / 2 \geq i+1, \ell_{0}+1 \neq(m+1) / 2$. Hence $w^{-1}\left(\Sigma_{i}\right) \cap \Gamma_{z}^{\prime} \neq \varnothing$, concluding the proof for $\boldsymbol{G}=\mathbf{S O}(2 n+1)$.

Assume now $\boldsymbol{G}=\mathbf{S O}(2 n)$; then we have $-\gamma=-e_{1}-e_{2}, \alpha_{j}=e_{j}-e_{j+1}$ for $j=1, \ldots, n-1$, and $\alpha_{n}=e_{n-1}+e_{n}$. Let $w$ be a Weyl element of $G$ such that $w^{-1}\left(\Sigma_{i}\right) \subset \Phi^{+}$; then, there is a permutation $\sigma$ of $\{1,2, \ldots, n\}$ and $\varepsilon_{1}, \varepsilon_{2} \in\{ \pm 1\}$ satisfying $\sigma(1)>\sigma(2)>\cdots>\sigma(i), \sigma(i+1)<\sigma(i+2)<\cdots<\sigma(n)$, $(-1)^{i-1} \varepsilon_{1} \varepsilon_{2}=1$, such that

$$
w^{-1}\left(e_{j}\right)=\left\{\begin{aligned}
\varepsilon_{1} e_{\sigma(1)} & \text { if } j=1 \\
-e_{\sigma(j)} & \text { if } 2 \leq j \leq i \\
e_{\sigma(j)} & \text { if } i+1 \leq j \leq n-1, \\
\varepsilon_{2} e_{\sigma(n)} & \text { if } j=n
\end{aligned}\right.
$$

Assume on the contrary that $w^{-1}\left(\Sigma_{i}\right) \cap \Gamma_{z}^{\prime}=\varnothing$; then

$$
w^{-1}\left(\Sigma_{i}\right) \subset \Gamma_{z} \text {. }
$$

By Lemma 2.8, $\lambda^{\prime}$ is of the form $\left[m, 2 n-m-2,1^{2}\right]$ or $[m, 2 n-m]$.

Assume first $m=2 n-3>\max (2 i-1,2 n-2 i-1), \lambda^{\prime}=\left[2 n-3,1^{3}\right]$; then $\Gamma_{z}$ is the set in (12). Since $i \in I_{\mathrm{nsp}}, I_{\mathrm{nsp}}$ is nonempty and $n \geq 4$. Hence 1,2,n-1,n are four distinct numbers. On the other hand, $\pm e_{1}-e_{2}, e_{n-1} \pm e_{n} \in \Sigma_{i}$, so by (23), $w^{-1}\left( \pm e_{1}-e_{2}\right)=e_{\sigma(2)} \pm \epsilon_{1} e_{\sigma(1)} \in \Gamma_{z}, \quad w^{-1}\left(e_{n-1} \pm e_{n}\right)=e_{\sigma(n-1)} \pm \epsilon_{2} e_{\sigma(n)} \in \Gamma_{z}$.

Hence the cardinality of $\{\sigma(1), \sigma(2), \sigma(n-1), \sigma(n)\}$ is 3 , which contradicts the assumption that $\sigma$ is a permutation.

Second, assume $\lambda^{\prime}=\left[m, 2 n-m-2,1^{2}\right]$ for some odd $m$ with $m\langle 2 n-3, m\rangle$ $\max (2 i-1,2 n-2 i-1)$. Then $\Gamma_{z}$ is the set in (13). Since $\pm e_{1}-e_{2}, e_{n-1} \pm e_{n} \in \Sigma_{i}$, we have, by (23),

$w^{-1}\left( \pm e_{1}-e_{2}\right)=e_{\sigma(2)} \pm \epsilon_{1} e_{\sigma(1)} \in \Gamma_{z}, \quad w^{-1}\left(e_{n-1} \pm e_{n}\right)=e_{\sigma(n-1)} \pm \epsilon_{2} e_{\sigma(n)} \in \Gamma_{z}$.

Then $\{\sigma(1), \sigma(n)\}=\{n-1, n\}$ and $\left\{\sigma(2), \sigma(n-1)=\{n-2, n-3\}\right.$. If $e_{2}-e_{3}$, $e_{n-2}-e_{n-1} \in \Sigma_{i}$, then by (23),

$w^{-1}\left(e_{2}-e_{3}\right)=e_{\sigma(3)}-e_{\sigma(2)} \in \Gamma_{z}, \quad w^{-1}\left(e_{n-2}-e_{n-1}\right)=e_{\sigma(n-2)}-e_{\sigma(n-1)} \in \Gamma_{z}$. 
Then $\{\sigma(3), \sigma(n-2)\}=\{n-5, n-4\}$. Since $m>2 i-1, m>2 n-2 i-1$,

$$
n-\frac{m+3}{2}<\min (i-1, n-i-1),
$$

the procedure can be repeated $n-\frac{m+3}{2}$ times. Then for $\ell=1,2, \ldots, n-\frac{m+1}{2}$,

$$
\{\sigma(\ell), \sigma(n+1-\ell)\}=\{n-2(\ell-1), n-2(\ell-1)-1\} .
$$

In particular, for $\ell_{0}=n-\frac{m+1}{2}$, we have $n+1-\ell_{0}=\frac{m+3}{2}$,

$$
\left\{\sigma\left(\ell_{0}\right), \sigma\left(n+1-\ell_{0}\right)\right\}=\left\{\sigma\left(\ell_{0}\right), \frac{m+3}{2}\right\}=\{m-n+3, m-n+2\} .
$$

Since $m>2 i-1$, we have $m>2 n-2 i-1$,

$\ell_{0}=n-\frac{m+1}{2}<i, \quad i+1<\frac{m+3}{2}=n+1-\ell_{0}, \quad e_{\ell_{0}}-e_{\ell_{0}+1}, e_{\frac{m+1}{2}}-e_{\frac{m+3}{2}} \in \Sigma_{i}$.

By (23),

$$
\begin{gathered}
w^{-1}\left(e_{\ell_{0}}-e_{\ell_{0}+1}\right)=e_{\sigma\left(\ell_{0}+1\right)}-e_{\sigma\left(\ell_{0}\right)} \in \Gamma_{z}, \\
w^{-1}\left(e_{\frac{m+1}{2}}-e_{\frac{m+3}{2}}\right)=e_{\sigma\left(\frac{m+1}{2}\right)}-e_{\sigma\left(\frac{m+3}{2}\right)} \in \Gamma_{z} .
\end{gathered}
$$

Then $\sigma\left(\ell_{0}+1\right)=\sigma\left(\frac{1}{2}(m+1)\right)=m-n+1$, which contradicts the assumption that $\sigma$ is a permutation, for $\ell_{0}+1 \leq i, \frac{1}{2}(m+1) \geq i+1, \ell_{0}+1 \neq \frac{1}{2}(m+1)$.

Third, assume $\lambda^{\prime}=[m, 2 n-m]$ for some odd $m \geq \max (2 i-1,2 n-2 i+1)$. Then $\Gamma_{z}$ is the set in (14). Since $\pm e_{1}-e_{2}, e_{n-1} \pm e_{n} \in \Sigma_{i}$, we have, by (23), $w^{-1}\left( \pm e_{1}-e_{2}\right)=e_{\sigma(2)} \pm \epsilon_{1} e_{\sigma(1)} \in \Gamma_{z}, \quad w^{-1}\left(e_{n-1} \pm e_{n}\right)=e_{\sigma(n-1)} \pm \epsilon_{2} e_{\sigma(n)} \in \Gamma_{z}$. Then $\sigma(1)=\sigma(n)=n$, which contradicts the assumption that $\sigma$ is a permutation.

Fourth, assume $n$ is even and $\lambda^{\prime}=\left[n^{2}\right]$. Then $\Gamma_{z}$ is either the set in (16) or the set in (17). Since $\pm e_{1}-e_{2}, e_{n-1} \pm e_{n}$ belong to $\Sigma_{i}$, by (23),

$w^{-1}\left( \pm e_{1}-e_{2}\right)=e_{\sigma(2)} \pm \epsilon_{1} e_{\sigma(1)} \in \Gamma_{z}, \quad w^{-1}\left(e_{n-1} \pm e_{n}\right)=e_{\sigma(n-1)} \pm \epsilon_{2} e_{\sigma(n)} \in \Gamma_{z}$.

Then $\sigma(1)=\sigma(n)=n$, which contradicts the assumption that $\sigma$ is a permutation. Hence $w^{-1}\left(\Sigma_{i}\right) \cap \Gamma_{z}^{\prime} \neq \varnothing$. This concludes the proof for $\boldsymbol{G}=\mathbf{S O}(2 n)$.

Assume now $\boldsymbol{G}=\mathbf{S p}(2 n)$; then we have $-\gamma=-2 e_{1}, \alpha_{j}=e_{j}-e_{j+1}$ for $j=1, \ldots, n-1$, and $\alpha_{n}=2 e_{n}$. Since $w^{-1}\left(\Sigma_{i}\right) \subset \Phi^{+}$, there is a permutation $\sigma$ of $\{1,2, \ldots, n\}$, satisfying $\sigma(1)>\sigma(2)>\cdots>\sigma(i), \sigma(i+1)<\sigma(i+2)<\cdots<\sigma(n)$, such that

$$
w^{-1}\left(e_{j}\right)=\left\{\begin{aligned}
-e_{\sigma(j)} & \text { if } 1 \leq j \leq i, \\
e_{\sigma(j)} & \text { if } i+1 \leq j \leq n .
\end{aligned}\right.
$$

By Lemma $2.8, \lambda^{\prime}=[m, 2 n-m]$ for some even $m>\max (2 i, 2 n-2 i)$. Then $\Gamma_{z}$ 
is the set in (18). Assume on the contrary that $w^{-1}\left(\Sigma_{i}\right) \cap \Gamma_{z}^{\prime}=\varnothing$; then

$$
w^{-1}\left(\Sigma_{i}\right) \subset \Gamma_{z} .
$$

Since $-2 e_{1}, 2 e_{n} \in \Sigma_{i}$, we have

$$
w^{-1}\left(-2 e_{1}\right)=2 e_{\sigma(1)} \in \Gamma_{z}, \quad w^{-1}\left(2 e_{n}\right)=2 e_{\sigma(n)} \in \Gamma_{z} .
$$

Then $\{\sigma(1), \sigma(n)\}=\{n-1, n\}$. If $e_{1}-e_{2}, e_{n-1}-e_{n} \in \Sigma_{i}$,

$w^{-1}\left(e_{1}-e_{2}\right)=e_{\sigma(2)}-e_{\sigma(1)} \in \Gamma_{O^{\prime}}, \quad w^{-1}\left(e_{n-1}-e_{n}\right)=e_{\sigma(n-1)}-e_{\sigma(n)} \in \Gamma_{O^{\prime}}$.

Then $\{\sigma(2), \sigma(n-1)\}=\{n-3, n-2\}$. Since $m>2 i$ and $m>2 n-2 i$, we have

$$
n-\frac{m}{2}<\max (i, n-i),
$$

the above procedure can be repeated $n-\frac{m}{2}$ times. Then for $\ell=1,2, \ldots, n-\frac{m}{2}$,

$$
\{\sigma(\ell), \sigma(n+1-\ell)\}=\{n-2(\ell-1), n-2(\ell-1)-1\} .
$$

In particular, for $\ell_{0}=n-\frac{m}{2}$ and $n+1-\ell_{0}=\frac{m}{2}+1$, we have

$$
\left\{\sigma\left(\ell_{0}\right), \sigma\left(n+1-\ell_{0}\right)\right\}=\left\{\sigma\left(\ell_{0}\right), \sigma\left(\frac{m}{2}+1\right)\right\}=\{m-n+1, m-n+2\} .
$$

Since $m>2 i, m>2 n-2 i$,

$\ell_{0}=n-\frac{m}{2}<i, \quad i+1<\frac{m}{2}+1=n+1-\ell_{0}, \quad e_{\ell_{0}}-e_{\ell_{0}+1}, e_{\frac{m}{2}}-e \frac{m}{2}+1 \in \Sigma_{i}$.

By assumption,

$$
\begin{aligned}
& w^{-1}\left(e_{\ell_{0}}-e_{\ell_{0}+1}\right)=e_{\sigma\left(\ell_{0}+1\right)}-e_{\sigma\left(\ell_{0}\right)} \in \Gamma_{O^{\prime}}, \\
& w^{-1}\left(e_{\frac{m}{2}}-e_{\frac{m}{2}+1}\right)=e_{\sigma\left(\frac{m}{2}\right)}-e_{\sigma\left(\frac{m}{2}+1\right)} \in \Gamma_{O^{\prime}} .
\end{aligned}
$$

Then $\sigma\left(\ell_{0}+1\right)=\sigma(m / 2)=m-n$. But $i \geq \ell_{0}+1 \neq m / 2>i$, which contradicts the assumption that $\sigma$ is a permutation. Hence $w^{-1}\left(\Sigma_{i}\right) \subset \Phi^{+}$. This conclude the proof for $\boldsymbol{G}=\mathbf{S p}(2 n)$.

Let $A=A(S)$ be the apartment of $\mathscr{B}(G)$ defined by the maximal split torus $S$ of $G$; see Section 2B. Let $r$ be a positive integer. $F \subset A$ is called an $r$-facet if $F$ is connected and there is a finite subset $\Phi_{F}$ of $\Phi_{\mathrm{af}}$ such that

$$
\psi(x)=r \quad \text { for all } x \in F, \psi \in \Phi_{F} .
$$

Here $\Phi_{\text {af }}$ is the set of affine roots associated to $S$. For more details on $r$-facets, see [DeBacker 2002]. Since $r$ is integer, the $r$-facet is in fact the usual facet. 
Lemma 3.4. For $i \in I_{\mathrm{nsp}}$, let $w$ be a Weyl element satisfying $w^{-1}\left(\Sigma_{i}\right) \subset \Phi^{+}$. Let $O^{\prime}, O^{i}$ be nilpotent orbits in $\mathfrak{g}$ corresponding to $\left(\lambda^{\prime},\left(q_{j}^{\prime}\right)\right)$ or $\left(\lambda^{\prime}, \phi, \epsilon\right)$ and $\left(\lambda^{i},\left(q_{j}\right)\right)$ respectively, with $O^{\prime}>O^{i}$. Let $z \in O^{\prime}$ be the nilpotent element in (6), (8), (10), (15), and let $r>0$ a positive integer. Then there is an $r$-facet $F$ such that $y_{i} \in \partial F$ and

$$
\left(w N_{\geq 4}^{\prime} w^{-1} \cap G_{y_{i}, r}\right) G_{y_{i}, r+} \supset G_{F, r+} .
$$

Here $y_{i}$ is the vertex of the fundamental chamber $C$ defined in Section $2 B$ and $N_{\geq j}^{\prime}$ is the object defined in Section $2 F$ for any $\mathfrak{s l}_{2}$ triple $\left\{z, h, z^{\prime}\right\}$ attached to $z$ in $\mathfrak{g}$.

Proof. Let $\Gamma_{z} \subset \Phi^{+}$be the set defined in (7), (9), (11), (13), and set $\Gamma_{z}^{\prime}=\Phi^{+} \backslash \Gamma_{z}$. By Lemma 3.3, $w^{-1}\left(\Sigma_{i}\right) \cap \Gamma_{z}^{\prime} \neq \varnothing$. Take $\beta \in \Sigma_{i}$ such that $w^{-1}(\beta) \in \Gamma_{z}^{\prime}$ and, let $x_{\beta}$ be an arbitrary point in the apartment $\mathscr{A}$ such that $0<\beta\left(x_{\beta}\right)<\frac{1}{2}$ and $\alpha\left(x_{\beta}\right)=0$ for all $\alpha \in \Sigma_{i}$ distinct from $\beta$. Let $F$ be the smallest $r$-facet containing $x_{\beta}$. Then $y_{i} \in \partial F$ and $F$ satisfies the requirement of the lemma.

In fact, let $\Phi_{i}$ be the root subsystem generated by $\Sigma_{i}$ and $\Phi_{i}^{+}$the subset of positive roots of $\Phi_{i}$ generated by $\Sigma_{i}$. Then by definition

$$
\mathfrak{g}_{F, r+}:=\mathfrak{g}_{x_{\beta}, r+}=\left(\prod_{\substack{\delta \in \Phi_{i} \\ \delta\left(x_{\beta}\right)>\delta\left(y_{i}\right)}} u_{\delta, r}\right)+\mathfrak{g}_{y_{i}, r+} \subset \mathfrak{g}_{y_{i}, r} .
$$

Note that the following sets are the same:

$$
\begin{aligned}
\left\{\delta \in \Phi_{i} \mid \delta\left(x_{\beta}\right)>\delta\left(y_{i}\right)\right\} & =\left\{\delta \in \Phi_{i}^{+} \mid \delta-\beta \in \Phi_{i}^{+}\right\} \\
& =\left\{\delta \in \Phi_{i}^{+} \mid \delta \in \beta+\Phi_{i}^{+}\right\} \\
& =\left\{w(\alpha) \in \Phi_{i}^{+} \mid \alpha \in w^{-1}(\beta)+w^{-1}\left(\Phi_{i}^{+}\right)\right\} .
\end{aligned}
$$

By Lemma 3.3, $w^{-1}(\beta) \in \Gamma_{z}^{\prime}$; that is, the root space $\mathfrak{u}_{w^{-1}(\beta)} \subset \mathfrak{n}_{\geq 4}^{\prime}$. On the other hand, since $w^{-1}\left(\Sigma_{i}\right) \subset \Phi^{+}, w^{-1}\left(\Phi_{i}^{+}\right) \subset \Phi^{+}$. For all $\delta \in \Phi^{+}, \mathfrak{u}_{\delta} \in \mathfrak{n}_{\geq 0}^{\prime}$ (see Appendix), so $u_{\alpha} \subset \mathfrak{n}_{\geq 4}^{\prime}$ for all $\alpha \in \Phi^{+} \cap\left(w^{-1}(\beta)+w^{-1}\left(\Sigma_{i}\right)\right)$.

Hence $\mathfrak{g}_{F, r+} \subset w \mathfrak{n}_{\geq 4}^{\prime} w^{-1} \cap \mathfrak{g}_{y_{i}, r}+\mathfrak{g}_{y_{i}, r+}$, and thus

$$
\left(w N_{\geq 4}^{\prime} w^{-1} \cap G_{y_{i}, r}\right) G_{y_{i}, r+} \supset G_{F, r+} .
$$

Proposition 3.5. Let $\pi=\pi_{\chi_{\boldsymbol{\mu}} ; \boldsymbol{\mu}^{\prime}} \in \Pi^{\prime}(\varphi)$ be an irreducible representation defined in Section $2 D$ such that $i=i\left(\mu^{\prime}\right) \in I_{\text {nsp. }}$. Let $O^{\prime}, O^{i}$ be nilpotent orbits in $\mathfrak{g}$ corresponding to $\left(\lambda^{\prime},\left(q_{j}^{\prime}\right)\right)$ or $\left(\lambda^{\prime}, \phi, \epsilon\right)$ and $\left(\lambda^{i},\left(q_{j}\right)\right)$ respectively, with $O^{\prime}>O^{i}$. Let $z \in O^{\prime}$ be the nilpotent element in (6), (8), (10), (15), and let $N_{\geq j}^{\prime}$ be the object defined in Section $2 F$ for any $\mathfrak{s l}_{2}$ triple $\left\{z, h, z^{\prime}\right\}$ attached to $z$ in $\mathfrak{g}$.

Let $N^{\prime}=N_{\geq 2}^{\prime}$ and $\psi_{z}$ the character of $N^{\prime}$ defined in (3). Let $v$ be a representative of a double coset in $G_{y_{i}} \backslash G / N^{\prime}$ and $\psi_{z}^{v}$ the character of $v N^{\prime} v^{-1} \cap G_{y_{i}}$ defined as 
follows: for all $x \in v N^{\prime} v^{-1} \cap G_{y_{i}}$,

$$
\psi_{z}^{v}(x):=\psi_{z}\left(v^{-1} x v\right) .
$$

Let $r>0$ be a positive integer. Then there is an $r$-facet $F$ such that $y_{i} \in \partial F$ and

$$
\left(v N^{\prime} v^{-1} \cap G_{y_{i}, r}\right) G_{y_{i}, r+} / G_{y_{i}, r+} \supset G_{F, r+} / G_{y_{i}, r+},\left.\quad \psi_{z}^{v}\right|_{G_{F, r+}}=1 .
$$

Proof. Let $S, B$ be the split torus and the Borel subgroup of $G$ defined in Section 2B and $U$ the unipotent subgroup of $B$. Let $v$ be a representative of $G_{y_{i}} \backslash G / N^{\prime}$; then,

$$
v=w \cdot a \cdot u
$$

for some Weyl element $w$ of $G$ such that $w^{-1}\left(\Sigma_{i}\right) \subset \Phi^{+}, a \in S$, and $u \in U / N^{\prime}$, where $\Sigma_{i}$ is the set defined in Lemma 3.3 (see [Reeder 1997]).

Note that $a, u$ normalize $N^{\prime}$, and let $\psi^{\prime}=\psi_{z}^{a u}$, the character of $N^{\prime}$ defined in (25) with $v$ replaced by $a u$. By Lemma 3.4, there is an $r$-facet $F$ with $y_{i} \in \partial F$ such that

$$
\left(v N^{\prime} v^{-1} \cap G_{y_{i}, r}\right) G_{y_{i}, r+} \supset\left(w N_{\geq 4}^{\prime} w^{-1} \cap G_{y_{i}, r}\right) G_{y_{i}, r+} \supset G_{F, r+} .
$$

For all $x \in G_{F, r+}$,

$$
v^{-1} x v \in(a u)^{-1} w^{-1}\left[w N_{\geq 4}^{\prime} w^{-1}\right] w a u \subset(a u)^{-1} N_{\geq 4}^{\prime} a u=N_{\geq 4} .
$$

By the definition of $\psi_{z}, \psi_{z}^{v}(x)=\psi_{z}\left(v^{-1} x v\right)=1$.

We can now conclude the proof of Theorem 3.1. By the discreteness criterion in [DeBacker and Reeder 2010, Lemma 2.4],

$$
\chi(\pi):=\left\{x \in \mathscr{B}(G) \mid V_{\pi}^{G_{x, r+}} \neq 0\right\}=G \cdot y_{i},
$$

and the $G_{y_{i}, r} / G_{y_{i}, r+-m o d u l e} V_{\pi}^{G_{y_{i}}, r+}$ is cuspidal; i.e., for any $r$-facet $F$ with $y_{i} \in \partial F$,

$$
\left(V_{\pi}^{G_{y_{i}}, r+}\right)^{\mathrm{L} F}=0 .
$$

Here $\mathrm{L}^{F}=G_{F, r+} / G_{y_{i}, r+}$ and $V_{\pi}$ is the representation space of $\pi$.

Assume on the contrary $\operatorname{Hom}_{N^{\prime}}\left(\pi, \psi_{z}\right) \neq 0$. By the construction of $\pi$ in [Adler 1998], $\pi=c-\operatorname{Ind}_{G_{y_{i}}}^{G}(\Xi)$ for some irreducible representation $\Xi$ of $G_{y_{i}}$. Let $V_{\Xi}$ be the space of $\Xi$. Then

$$
\operatorname{Hom}_{N^{\prime}}\left(\pi, \psi_{z}\right)=\prod_{v \in G_{y_{i}} \backslash G / N^{\prime}} \operatorname{Hom}_{v N^{\prime} v^{-1} \cap G_{y_{i}}}\left(\Xi, \psi_{z}^{v}\right),
$$

and there is some $v \in G_{y_{i}} \backslash G / N^{\prime}$ such that $\operatorname{Hom}_{v N^{\prime} v^{-1} \cap G_{y_{i}}}\left(\Xi, \psi_{z}^{v}\right) \neq 0$. Then

$$
\operatorname{Hom}_{v N^{\prime} v^{-1} \cap G_{y_{i}, r}}\left(\Xi, \psi_{z}^{v}\right) \neq 0 .
$$


Applying Proposition 3.5, there is an $r$-facet $F$ such that $y_{i} \in \partial F$ and $V_{\Xi}^{G_{F, r+}} \neq 0$. Then $V_{\pi}^{G_{F, r+}} \neq 0$, which contradicts the discreteness criterion (26).

Proof of Theorem 3.2. Let $\overline{\mathfrak{f}}$ be the algebraic closure of $\mathfrak{f}$. Assume the characteristic $p$ of $\mathfrak{f}$ is large enough that $p$ is a good prime in the sense of [Carter 1972].

Keep the notation of Proposition 3.5. Then $i=i\left(\mu^{\prime}\right) \in I_{\mathrm{nsp}}$ and $G_{y_{i}, r} / G_{y_{i}, r+}=$ $\mathfrak{g}_{1}(\mathfrak{f}) \times \mathfrak{g}_{2}(\mathfrak{f})$, with $\mathfrak{g}_{1}=\mathfrak{s o}(2 i, f)$ or $\mathfrak{s p}(2 i, f)$ (see Section $\left.2 \mathrm{~B}\right)$. Let $\bar{\xi}_{j} \in \mathfrak{g}_{j}(\mathfrak{f})$ $(j=1,2)$ be regular nilpotent elements and $\left\{\bar{\xi}_{j}, \bar{h}_{j}, \bar{\xi}_{j}^{\prime}\right\}$ an $\mathfrak{s l}_{2}$ triple in $\mathfrak{g}_{j}(\mathfrak{f})$ attached to $\bar{\xi}_{j}$. Let

$$
\bar{\xi}=\left(\bar{\xi}_{1}, \bar{\xi}_{2}\right), \quad \bar{h}=\left(\bar{h}_{1}, \bar{h}_{2}\right), \quad \bar{\xi}^{\prime}=\left(\bar{\xi}_{1}, \bar{\xi}_{2}\right) .
$$

Then $\left(\bar{\xi}, \bar{h}, \bar{\xi}^{\prime}\right)$ is an $\mathfrak{s l}_{2}$ triple in $\mathfrak{g}_{1}(\mathfrak{f}) \times \mathfrak{g}_{2}(\mathfrak{f})$.

Recall that if $\boldsymbol{\mu}^{\prime} \in \mathscr{S}(\boldsymbol{\mu}), i=i_{\boldsymbol{\mu}^{\prime}} \in I_{\mathrm{nsp}}$, then $\mathrm{T}:=\mathrm{T}_{\boldsymbol{\mu}^{\prime}}=\mathrm{T}_{1} \times \mathrm{T}_{2}$ is a maximal anisotropic torus in $\mathrm{G}_{y_{i}}$. Let $T:=T_{\boldsymbol{\mu}^{\prime}}$ be the maximal anisotropic unramified torus in $G$ associated to $\left(y_{i}, \mathrm{~T}_{\boldsymbol{\mu}^{\prime}}\right)$ in Section 2C. Let $X=X_{\boldsymbol{\mu}^{\prime}} \in \mathfrak{t}=\operatorname{Lie}(T)$ be the good element of depth $-r$ defining $\pi_{\chi_{\mu} ; \boldsymbol{\mu}^{\prime}}$, whose image under the natural projection

$$
\mathfrak{g}_{y_{i},-r} \rightarrow \mathfrak{g}_{y_{i},-r} / \mathfrak{g}_{y_{i},-r+} \simeq \mathfrak{g}_{1} \times \mathfrak{g}_{2} .
$$

is denoted by $\bar{X}=\left(\bar{X}_{1}, \bar{X}_{2}\right)$. Since $X$ is a good element in $\mathfrak{t}$ with $C_{G}(X)=T$, $\bar{X}_{j}$ is a regular semisimple element in $\operatorname{Lie}\left(\mathrm{T}_{j}\right)(\mathfrak{f})$ for $j=1,2$.

Let $O_{\bar{X}_{j}}$ be the orbit of $\bar{X}_{j}$ in $\mathfrak{g}_{j}(\overline{\mathfrak{f}}) / \mathrm{G}_{j}(\overline{\mathfrak{f}})$. By [Slodowy 1980, §7.4, Corollary 2], the Slodowy slice

$$
\bar{V}_{j}:=\bar{\xi}_{j}+C_{\mathfrak{g}_{j}(\overline{\mathfrak{f}})}\left(\bar{\xi}_{j}^{\prime}\right)
$$

intersects $O_{\bar{X}_{j}}$ at a unique $\mathfrak{f}$-rational point $\bar{X}_{j}^{\prime} \in \mathfrak{g}_{j}(\mathfrak{f})$.

Since $X$ is good, $C_{\mathrm{G}_{j}(\overline{\mathfrak{f}})}\left(\bar{X}_{j}\right)$ is connected [Carter 1985, Theorem 3.5.3]. Then there is a $\bar{g}_{j} \in \mathrm{G}_{j}(\mathfrak{f})$ such that $\operatorname{Ad}\left(\bar{g}_{j}\right)\left(X_{j}\right)=\bar{X}_{j}^{\prime}$ [Digne and Michel 1991, §3.25]. Moreover $\mathrm{T}_{j}^{\prime}=C_{\mathrm{G}_{j}}\left(\bar{X}_{j}^{\prime}\right)=\operatorname{Ad}\left(\bar{g}_{j}\right)\left(\mathrm{T}_{j}\right)$ is a maximal anisotropic torus of $\mathrm{G}_{j}(\mathfrak{f})$, with $\mathrm{G}_{j}(\mathfrak{f})$-conjugate to $\mathrm{T}_{j}$. Let $\bar{g}=\left(\bar{g}_{1}, \bar{g}_{2}\right) \in \mathrm{G}(\mathfrak{f})$; then, $\operatorname{Ad}(\bar{g})\left(\mathrm{T}_{1} \times \mathrm{T}_{2}\right)=\mathrm{T}^{\prime}:=$ $\mathrm{T}_{1}^{\prime} \times \mathrm{T}_{2}^{\prime}$.

Let $g \in G_{y_{i}, 0}-g_{y_{i}, 0+}$ such that $g$ projects to $\bar{g}, T^{\prime}:=\operatorname{Ad}(g)(T)$, and $X^{\prime}:=$ $\operatorname{Ad}(g)(X) \in \mathfrak{t}^{\prime}$. Then $T^{\prime}$ is the maximal unramified torus in $G$, associated to $\left(y_{i}, \mathrm{~T}^{\prime}\right)$, $X^{\prime}$ is a good element in $\mathfrak{g}_{y_{i},-r} \backslash \mathfrak{g}_{y_{i},-r+}$, whose image under the natural projection in $\mathrm{G}_{y_{i}}$ is $\bar{X}^{\prime}=\left(\bar{X}_{1}^{\prime}, \bar{X}_{2}^{\prime}\right)$. Note that $\bar{X}^{\prime} \in \bar{V}_{1}(\mathfrak{f}) \times \bar{V}_{2}(\mathfrak{f})$, where

$$
\bar{V}_{1}(\mathfrak{f})=\bar{\xi}_{1}+C_{\mathfrak{g}_{1}(\mathfrak{f})}\left(\bar{\xi}_{1}^{\prime}\right), \quad \bar{V}_{2}(\mathfrak{f})=\bar{\xi}_{2}+C_{\mathfrak{g}_{2}(\mathfrak{f})}\left(\bar{\xi}_{2}^{\prime}\right)
$$

are sets of $\mathfrak{f}$-rational points of $\bar{V}_{1}, \bar{V}_{2}$ respectively. Without loss of generality, assume $X=X^{\prime}$. Then the natural image $\bar{X}$ of $X$ in $\mathfrak{g}_{y_{i},-r} / \mathfrak{g}_{y_{i},-r+}$ belongs to $\bar{V}_{1}(\mathfrak{f}) \times \bar{V}_{2}(\mathfrak{f})$. 
By [DeBacker 2002, Corollary 4.3.2], let $\left(\xi, h, \xi^{\prime}\right) \in \mathfrak{g}_{y_{i},-r} \times \mathfrak{g}_{y_{i}, 0} \times \mathfrak{g}_{y_{i}, r}$ be an $\mathfrak{s l}_{2}$ triple in $\mathfrak{g}$ such that $\left\{\xi, h, \xi^{\prime}\right\}$ lifts $\left\{\bar{\xi}, \bar{h}, \bar{\xi}^{\prime}\right\}$ respectively and $O^{\prime}=\operatorname{Ad}(G)(\xi)$ the nilpotent orbit of $\xi$ in $\mathfrak{g}$. By the choice of $\left\{\xi, h, \xi^{\prime}\right\}, O^{\prime}=O^{i}$ is a nilpotent orbit corresponding to $\left(\lambda^{i},\left(q_{j}\right)\right)$. Let $N_{\geq j}^{\prime}$ be the object defined in Section $2 \mathrm{~F}$ for the triple $\left\{\xi, h, \xi^{\prime}\right\}$ attached to $\xi$ in $\mathfrak{g}$.

We can now conclude the proof of Theorem 3.2. Let $N^{\prime}=N_{\geq 2}^{\prime}$ and let $S_{\xi}$ be the character $\psi_{\xi}$ of $N^{\prime}$ :

$$
S_{\xi}(\exp Y)=\psi \circ \operatorname{tr}(\xi Y), \quad Y \in \operatorname{Lie}\left(N^{\prime}\right) .
$$

On the other hand, by the construction in [Adler 1998], $\pi_{\chi_{\boldsymbol{\mu}} ; \boldsymbol{\mu}^{\prime}}=c-\operatorname{Ind}_{G_{y_{i}}}^{G(k)}(\Xi)$, while $\Xi=\operatorname{Ind}_{T J}^{G_{y_{i}}}\left(\sigma_{\chi}\right)$. Here

$$
\begin{aligned}
J & =\exp _{y_{i}}(\mathfrak{J}), \quad \mathfrak{J}=\mathfrak{t}_{y_{i}, r}+\mathfrak{t}_{y_{i}, \frac{r}{2}}^{\perp}, \\
J^{+} & =\exp _{y_{i}}\left(\mathfrak{J}^{+}\right), \quad \mathfrak{J}^{+}=\mathfrak{t}_{y_{i}, r}+\mathfrak{t}_{y_{i}, \frac{r}{2}+}^{\perp},
\end{aligned}
$$

with $\mathfrak{t}^{\perp}$ the orthogonal complement of $\mathfrak{t}$ in $\mathfrak{g}$ with respect to the killing form. Here $T J$ and $T J^{+}$are subgroups of $G$, since $T$ normalizes $J$ and $J^{+}$, and $\sigma_{\chi}$ is the irreducible representation of $T J$ such that $\left.\sigma_{\chi}\right|_{T J^{+}}$is a multiple of $\chi$, where $\chi$ is the character of $T J^{+}$extending $\chi_{\boldsymbol{\mu}^{\prime}}$ on $T$, such that

$$
\chi\left(\exp _{y_{i}} Y\right)=\psi(\operatorname{tr}(X \cdot Y)) \text { for all } Y \in \mathfrak{J}^{+} .
$$

Note that $T$ is anisotropic and $N^{\prime} \cap T J=N^{\prime} \cap J \supset N^{\prime} \cap J^{+}$, while $N^{\prime} \cap J / N^{\prime} \cap J^{+}$ is an isotropic subspace over $\mathfrak{f}$ with respect to the nondegenerate symplectic form defined on $J / J^{+}$by $\left(n, n^{\prime}\right) \mapsto \psi_{\xi}\left(\left[\log n, \log n^{\prime}\right]\right)$. On the other hand, since $\bar{X} \in$ $\bar{V}_{1}(\mathfrak{f}) \times \bar{V}_{2}(\mathfrak{f}),\left.\chi\right|_{J+\cap N^{\prime}}=\left.\psi_{\xi}\right|_{J^{+} \cap N^{\prime}}$. By the definition of $\sigma_{\chi}$,

$$
\operatorname{Hom}_{N^{\prime} \cap T J}\left(\sigma_{\chi}, \psi_{\xi}\right)=\operatorname{Hom}_{N^{\prime} \cap J}\left(\sigma_{\chi}, \psi_{\xi}\right) \neq 0 .
$$

Apply Lemma 3.6 below with $G_{1}$ replaced by $G_{y_{i}}, G_{2}$ by $N^{\prime} \cap G_{y_{i}}$, and $H_{1}$ by $T J$; then,

$$
\operatorname{Hom}_{N^{\prime} \cap G_{y_{i}}}\left(\Xi, \psi_{\xi}\right) \neq 0 .
$$

Since $\operatorname{Hom}_{N^{\prime}}\left(\pi_{\chi_{\boldsymbol{\mu}} ; \boldsymbol{\mu}^{\prime}}, S_{\xi}\right)=\prod_{v \in G_{y_{i}} \backslash G / N^{\prime}} \operatorname{Hom}_{v N^{\prime} v^{-1} \cap G_{y_{i}}}\left(\Xi, \psi_{\xi}^{v}\right)$, by (28),

$$
\operatorname{Hom}_{N^{\prime}}\left(\pi_{\chi_{\boldsymbol{\mu}} ; \boldsymbol{\mu}^{\prime}}, \psi_{\xi}\right) \neq 0 .
$$

Hence $O^{\prime} \in \mathcal{N}_{\text {wh }}\left(\pi_{\chi_{\boldsymbol{\mu}} ; \boldsymbol{\mu}^{\prime}}\right)$. Combining with Theorem 3.1, $O^{\prime} \in \mathcal{N}_{\text {wh,max }}\left(\pi_{\chi_{\boldsymbol{\mu}} ; \boldsymbol{\mu}^{\prime}}\right)$.

Lemma 3.6. Let $G_{1}$ be a compact subgroup, and $H_{1}, G_{2}$ open compact subgroups of $G_{1}$. Let $\left(\sigma, V_{\sigma}\right)$ (resp. $\left.\left(\xi, V_{\xi}\right)\right)$ be a smooth representation of $H_{1}$ (resp. $\left.G_{2}\right)$. If $\operatorname{Hom}_{H_{1} \cap G_{2}}(\sigma, \xi) \neq 0$, then $\operatorname{Hom}_{G_{2}}\left(\operatorname{Ind}_{H_{1}}^{G_{1}} \sigma, \xi\right) \neq 0$. 
Proof. The proof is similar to that of Proposition 2.1 in [Arthur 2008]. Consider a nonzero $A \in \operatorname{Hom}_{H_{1} \cap G_{2}}(\sigma, \xi)$, and define $J_{A} \in \operatorname{Hom}_{G_{2}}\left(\operatorname{Ind}_{H_{1}}^{G_{1}} \sigma, \xi\right)$ as follows: for arbitrary $\phi \in \operatorname{Ind}_{H_{1}}^{G_{1}} \sigma$,

$$
J_{A} \phi=\sum_{H_{1} \cap G_{2} \backslash G_{2}} \xi\left(g^{\prime}\right)^{-1} A\left(\phi\left(g^{\prime}\right)\right) \in V_{\xi}
$$

For all $g \in G_{2}$,

$$
\begin{aligned}
J_{A}(\operatorname{Ind} \sigma)(g) \phi & =\sum_{H_{1} \cap G_{2} \backslash G_{2}} \xi\left(g^{\prime}\right)^{-1} A(\operatorname{Ind} \sigma(g) \phi)\left(g^{\prime}\right) \\
& =\sum_{H_{1} \cap G_{2} \backslash G_{2}} \xi\left(g^{\prime}\right)^{-1} A \phi\left(g^{\prime} g\right) \\
& =\xi(g) J_{A} \phi .
\end{aligned}
$$

Take some $v \in V_{\sigma}$ such that $A v \neq 0$. Define $\phi_{v}(g)=\sigma(h) v$ if $g=h \in H_{1}$ and $\phi_{v}(g)=0$ if $g \notin H_{1}$. Then $\phi_{v} \in \operatorname{Ind}_{H_{1}}^{G_{1}} \sigma$, and $J_{A} \phi_{v}=A v \neq 0$, so $J_{A}$ is a nonzero element in $\operatorname{Hom}_{G_{2}}\left(\operatorname{Ind}_{H_{1}}^{G_{1}} \sigma, \xi\right)$.

\section{Appendix: Rational nilpotent orbits}

In this section, we show by example how to choose a particular element from a rational nilpotent orbit parametrized by $\left(\lambda,\left(q_{j}\right)\right)$.

Let $W$ be a $(2 n+1)$-dimensional symmetric $k$-space as defined in Section 2A, with bilinear form $q_{W}$. Let $z$ be a nonzero nilpotent element in $\mathfrak{g}=\mathfrak{s o}(W) \subset \mathfrak{g l}(W)$, and set $G=\mathbf{S O}(k, W)$. Let $\phi: \mathfrak{s l}_{2} \rightarrow \mathfrak{g}$ be a Lie algebra homomorphism with

$$
\phi\left(\left(\begin{array}{ll}
0 & 0 \\
1 & 0
\end{array}\right)\right)=z .
$$

Identify a scalar $t \in k$ with the diagonal matrix $\operatorname{diag}\left(t, t^{-1}\right) \in \mathfrak{s l}_{2}(k)$. As in [Mœglin 1996], for $i \in \mathbb{Z}$, let

$$
\begin{aligned}
\mathfrak{g}(i) & =\{Y \in \mathfrak{g} \mid \operatorname{Ad} \circ \phi(t)(Y)=i t Y \text { for all } t \in k\}, \\
W(i) & =\{v \in W \mid \phi(t)(v)=i t v \text { for all } t \in k\} .
\end{aligned}
$$

Then $\mathfrak{g}=\bigoplus_{i \in \mathbb{Z}} \mathfrak{g}(i), W=\bigoplus_{i \in \mathbb{Z}} W(i)$.

Assume the orbit $O=\operatorname{Ad}(G)(z)$ of $z$ is parametrized by $\left(\lambda,\left(q_{i}\right)\right)$ with $\lambda=$ [ $m, 2 n-m, 1]$, where $m>n$ is an odd number. For $i=1, \ldots, 2 n+1$, let

$$
W_{i}=\operatorname{Ker}\left(z^{i}\right) /\left(\operatorname{Ker}\left(z^{i-1}\right)+z \operatorname{Ker}\left(z^{i+1}\right)\right) .
$$


Then by [Waldspurger 2001, §I.6], $\operatorname{dim} W_{i}=c_{i}(\boldsymbol{\lambda})$ and $q_{i}$ is the nondegenerate quadratic form on $W_{i}$ defined by

$$
q_{i}\left(\bar{v}, \bar{v}^{\prime}\right)=(-1)^{\left[\frac{i-1}{2}\right]} q_{W}\left(z^{i-1} v, v^{\prime}\right) \quad\left(\bar{v}, \bar{v}^{\prime} \in W_{i}\right),
$$

where $v$ (resp. $\left.v^{\prime}\right)$ is an inverse image of $\bar{v}\left(\right.$ resp. $\left.\bar{v}^{\prime}\right)$ in $\operatorname{Ker}\left(z^{i}\right)$.

Assume $m=2 n-1$; in this case $\lambda=\left[2 n-1,1^{2}\right], c_{1}(\lambda)=2, c_{2 n-1}(\lambda)=1$. Then $\operatorname{dim} W_{1}=2$ and $\operatorname{dim} W_{m}=1$. By (29), let $v_{1}, v_{1}^{\prime} \in \operatorname{Ker} z, v_{m} \in \operatorname{Ker} z^{m}$ such that

$$
\begin{aligned}
\operatorname{Ker} z & =z \operatorname{Ker} z^{2} \oplus k v_{1} \oplus k v_{1}^{\prime}, \\
\operatorname{Ker} z^{m} & =\left(\operatorname{Ker} z^{m-1}+z \operatorname{Ker} z^{m+1}\right) \oplus k v_{m} .
\end{aligned}
$$

Let $\bar{v}_{1}, \bar{v}_{1}^{\prime}$ be the natural images of $v_{1}, v_{1}^{\prime}$ in $W_{1}$ and $\bar{v}_{m}$ that of $v_{m}$ in $W_{m}$. Without loss of generality, assume $\bar{v}_{1}, \bar{v}_{1}^{\prime}$ are orthogonal to each other under $q_{1}$; then $q_{1}=\left\langle q_{1}\left(\bar{v}_{1}, \bar{v}_{1}\right), q_{1}\left(\bar{v}_{1}^{\prime}, \bar{v}_{1}^{\prime}\right)\right\rangle$,

$$
q_{m}=\left\langle q_{m}\left(\bar{v}_{m}, \bar{v}_{m}\right)\right\rangle=(-1)^{\frac{m-1}{2}} q_{W}\left(z^{m-1} v_{m}, v_{m}\right) .
$$

In the following, identify $q_{m}$ with $q_{m}\left(\bar{v}_{m}, \bar{v}_{m}\right)$.

Through $\phi: \mathfrak{s l}_{2} \rightarrow \mathfrak{s o}(W) \subset \mathfrak{g l}(W), W$ is a representation space of $\mathfrak{s l}_{2}$. In fact, since $O_{X}$ corresponds to $\left(\lambda,\left(q_{i}\right)\right), W \simeq V_{m} \oplus V_{1} \oplus V_{1}$, where $V_{j}$ is the irreducible representation of $\mathfrak{s l}_{2}$ of dimension $j$. By the representation theory of $\mathfrak{s l}_{2}, v_{1}, v_{1}^{\prime} \in W(0)$ and $v_{m} \in W(m-1)$. Modifying by elements in $z \operatorname{Ker} z^{2}$, we can assume further that the subspace generated by $v_{1}, v_{1}^{\prime}$ is $V_{1} \oplus V_{1}$.

Then $0 \neq z^{\ell}\left(v_{m}\right) \in W(m-1-2 \ell)$ for all $\ell=1, \ldots, m-1$, and

$$
\begin{aligned}
W(m-1) & =k v_{m}, \\
W(m-3) & =k z v_{m}, \\
\vdots & \vdots \\
W(2) & =k z^{n-2} v_{m}, \\
W(0) & =k z^{n-1} v_{m} \oplus k v_{1} \oplus k v_{1}^{\prime}, \\
W(-2) & =k z^{n} v_{m}, \\
\vdots & \vdots \\
W(-(m-1)) & =k z^{m-1} v_{m} .
\end{aligned}
$$

For $j=1, \ldots, m$, let $F_{j}=\bigoplus_{\ell \leq-(m-1)+2(j-1)} W(\ell)$ be a subspace of $W$. Then

$$
0=F_{0} \subset F_{1} \subset F_{2} \subset \cdots \subset F_{m}=W
$$


and $z F_{j}=F_{j-1}$ for $j=1, \ldots, m$. Take a basis of $W$ such that

$$
\begin{aligned}
e_{1} & =v_{m}, & e_{-1} & =(-1)^{\frac{m-1}{2}} q_{m}^{-1} z^{m-1} v_{m}, \\
e_{2} & =z v_{m}, & e_{-2} & =(-1)^{\frac{m-3}{2}} q_{m}^{-1} z^{m-2} v_{m}, \\
& \vdots & \vdots & \\
e_{n-1} & =z^{n-2} v_{m}, & e_{-(n-1)} & =(-1) q_{m}^{-1} z^{n+1} v_{m} .
\end{aligned}
$$

By (30), $q_{W}\left(e_{i}, e_{j}\right)=0$ unless $i+j=0$, and $q_{W}\left(e_{i}, e_{-i}\right)=1$. Note that $W(0)$ has orthogonal decomposition

$$
W(0)=k z^{n-1} v_{m} \oplus k v_{1} \oplus k v_{1}^{\prime}
$$

under $\left.q_{W}\right|_{W(0)}$. By (30), $q_{W}\left(z^{n-1} v_{m}, z^{n-1} v_{m}\right)=q_{m}\left(\bar{v}_{m}, \bar{v}_{m}\right), q_{W}\left(v_{1}, v_{1}\right)=$ $q_{1}\left(\bar{v}_{1}, \bar{v}_{1}\right)$, and $q_{W}\left(v_{1}^{\prime}, v_{1}^{\prime}\right)=q_{1}\left(\bar{v}_{1}^{\prime}, \bar{v}_{1}^{\prime}\right)$. By $(31)$,

$$
\begin{aligned}
\left.q_{W}\right|_{W(0)} & =\left\langle q_{W}\left(z^{\frac{m-1}{2}} v_{m}, z^{\frac{m-1}{2}} v_{m}\right), q_{W}\left(v_{1}, v_{1}\right), q_{W}\left(v_{1}^{\prime}, v_{1}^{\prime}\right)\right\rangle \\
& =\left\langle q_{m}\left(\bar{v}_{m}, \bar{v}_{m}\right), q_{1}\left(\bar{v}_{1}, \bar{v}_{1}\right), q_{1}\left(\bar{v}_{1}^{\prime}, \bar{v}_{1}^{\prime}\right)\right\rangle \\
& =q_{m} \oplus q_{1} .
\end{aligned}
$$

Because $q_{1} \oplus q_{m}$ has the same anisotropic kernel as $W$, let $e_{n}, e_{0}, e_{-n}$ be a basis of $W(0)$ such that

$$
q_{W}\left(e_{n}, e_{-n}\right)=1, \quad q_{W}\left(e_{0}, e_{0}\right)=1, \quad q_{W}\left(e_{n}, e_{0}\right)=q_{W}\left(e_{-n}, e_{0}\right)=0 .
$$

Then $e_{1}, e_{2}, \ldots, e_{n}, e_{0}, e_{-n}, \ldots, e_{-1}$ is a basis of $W$, under which the matrix of $q_{W}$ is $J_{W}$ (defined in Section 2A), and the matrix of $z$ is the lower triangular block matrix

$$
\left(\begin{array}{cccccccc}
0 & & & & & & & \\
1 & 0 & & & & & & \\
& \ddots & \ddots & & & & & \\
& & 1 & 0 & & & & \\
& & & A^{*} & 0_{3} & & & \\
& & & & A & 0 & & \\
& & & & & -1 & 0 & \\
& & & & & & \ddots & \ddots
\end{array}\right),
$$

with

$$
A^{*}=\left(\begin{array}{l}
a_{m} \\
b_{m} \\
c_{m}
\end{array}\right), \quad A=-\left(\begin{array}{lll}
c_{m} & b_{m} & a_{m}
\end{array}\right),
$$

where $\left(a_{m}, b_{m}, c_{m}\right)$ are the coordinates of $z^{n-1} v_{m}$ in the basis $\left\{e_{n}, e_{0}, e_{-n}\right\}$. Note that in this case, $A A^{*}=-q_{m}$. 


\section{Acknowledgments}

The author would like to thank the referee for carefully reading and giving good advice on organizing and revising this paper. This paper was started and completed during a visit to University of Toronto in 2011. The author also thanks Professor Murnaghan for her hospitality and significant discussions during the visit.

\section{References}

[Adler 1998] J. D. Adler, "Refined anisotropic $K$-types and supercuspidal representations", Pacific J. Math. 185:1 (1998), 1-32. MR 2000f:22019 Zbl 0924.22015

[Arthur 2008] J. Arthur, "Induced representations, intertwining operators and transfer", pp. 51-67 in Group representations, ergodic theory, and mathematical physics: a tribute to George W. Mackey (New Orleans, LA, 2007), edited by R. S. Doran et al., Contemp. Math. 449, American Mathematical Society, Providence, RI, 2008. MR 2009c:22015 Zbl 1158.22016

[Barbasch and Moy 1997] D. Barbasch and A. Moy, "Local character expansions", Ann. Sci. École Norm. Sup. (4) 30:5 (1997), 553-567. MR 99j:22021 Zbl 0885.22021

[Carter 1972] R. W. Carter, "Conjugacy classes in the Weyl group", Compositio Math. 25 (1972), 1-59. MR 47 \#6884 Zbl 0254.17005

[Carter 1985] R. W. Carter, Finite groups of Lie type: conjugacy classes and complex characters, Wiley, New York, 1985. MR 87d:20060 Zbl 0567.20023

[Collingwood and McGovern 1993] D. H. Collingwood and W. M. McGovern, Nilpotent orbits in semisimple Lie algebras, Van Nostrand Reinhold, New York, 1993. MR 94j:17001 Zbl 0972.17008

[DeBacker 2002] S. DeBacker, "Parametrizing nilpotent orbits via Bruhat-Tits theory", Ann. of Math.

(2) 156:1 (2002), 295-332. MR 2003i:20086 Zbl 1015.20033

[DeBacker 2006] S. DeBacker, "Parameterizing conjugacy classes of maximal unramified tori via Bruhat-Tits theory", Michigan Math. J. 54:1 (2006), 157-178. MR 2007d:22012 Zbl 1118.22005

[DeBacker and Reeder 2010] S. DeBacker and M. Reeder, "On some generic very cuspidal representations”, Compositio Math. 146:4 (2010), 1029-1055. MR 2011j:20114 Zbl 1195.22011

[Digne and Michel 1991] F. Digne and J. Michel, Representations of finite groups of Lie type, London Mathematical Society Student Texts 21, Cambridge University Press, 1991. MR 92g:20063 Zbl 0815.20014

[Mœglin 1996] C. Mœglin, "Front d'onde des représentations des groupes classiques $p$-adiques", Amer. J. Math. 118:6 (1996), 1313-1346. MR 98d:22015 Zbl 0864.22007

[Mœglin and Waldspurger 1987] C. Mœglin and J.-L. Waldspurger, "Modèles de Whittaker dégénérés pour des groupes p-adiques", Math. Z. 196:3 (1987), 427-452. MR 89f:22024 Zbl 0612.22008

[Reeder 1997] M. Reeder, "Whittaker models and unipotent representations of $p$-adic groups", Math. Ann. 308:4 (1997), 587-592. MR 98h:22022 Zbl 0874.22015

[Reeder 2008] M. Reeder, "Supercuspidal $L$-packets of positive depth and twisted Coxeter elements", J. Reine Angew. Math. 620 (2008), 1-33. MR 2009e:22019 Zbl 1153.22021

[Slodowy 1980] P. Slodowy, Simple singularities and simple algebraic groups, Lecture Notes in Math. 815, Springer, Berlin, 1980. MR 82g:14037 Zbl 0441.14002

[Tits 1979] J. Tits, "Reductive groups over local fields", pp. 29-69 in Automorphic forms, representations and L-functions (Corvallis, OR, 1977), vol. 1, edited by A. Borel and W. Casselman, Proc. Sympos. Pure Math. 33, American Mathematical Society, Providence, RI, 1979. MR 80h:20064 Zbl 0415.20035 
[Waldspurger 2001] J.-L. Waldspurger, Intégrales orbitales nilpotentes et endoscopie pour les groupes classiques non ramifiés, Astérisque 269, Société Mathématique de France, Paris, 2001. MR 2002h:22014 Zbl 0965.22012

Received October 25, 2012. Revised March 23, 2014.

QIN YUJUN

DEPARTMENT OF MATHEMATICS

EAST CHINA NORMAL UNIVERSITY

DONGCHUAN ROAD 500

SHANGHAI 200241

CHINA

yjqin@math.ecnu.edu.cn 


\title{
PACIFIC JOURNAL OF MATHEMATICS
}

\author{
msp.org/pjm
}

Founded in 1951 by E. F. Beckenbach (1906-1982) and F. Wolf (1904-1989)

\section{EDITORS}

Don Blasius (Managing Editor)

Department of Mathematics

University of California

Los Angeles, CA 90095-1555

blasius@math.ucla.edu

\author{
Paul Balmer \\ Department of Mathematics \\ University of California \\ Los Angeles, CA 90095-1555 \\ balmer@math.ucla.edu \\ Robert Finn \\ Department of Mathematics \\ Stanford University \\ Stanford, CA 94305-2125 \\ finn@math.stanford.edu \\ Sorin Popa \\ Department of Mathematics \\ University of California \\ Los Angeles, CA 90095-1555 \\ popa@math.ucla.edu
}

\author{
Vyjayanthi Chari \\ Department of Mathematics \\ University of California \\ Riverside, CA 92521-0135 \\ chari@math.ucr.edu \\ Kefeng Liu \\ Department of Mathematics \\ University of California \\ Los Angeles, CA 90095-1555 \\ liu@math.ucla.edu \\ Jie Qing \\ Department of Mathematics \\ University of California \\ Santa Cruz, CA 95064 \\ qing@ cats.ucsc.edu
}

\section{PRODUCTION}

Silvio Levy, Scientific Editor, production@msp.org

\section{SUPPORTING INSTITUTIONS}

ACADEMIA SINICA, TAIPEI

CALIFORNIA INST. OF TECHNOLOGY

INST. DE MATEMÁTICA PURA E APLICADA

KEIO UNIVERSITY

MATH. SCIENCES RESEARCH INSTITUTE

NEW MEXICO STATE UNIV.

OREGON STATE UNIV.

\author{
STANFORD UNIVERSITY \\ UNIV. OF BRITISH COLUMBIA \\ UNIV. OF CALIFORNIA, BERKELEY \\ UNIV. OF CALIFORNIA, DAVIS \\ UNIV. OF CALIFORNIA, LOS ANGELES \\ UNIV. OF CALIFORNIA, RIVERSIDE \\ UNIV. OF CALIFORNIA, SAN DIEGO \\ UNIV. OF CALIF., SANTA BARBARA
}

\author{
Daryl Cooper \\ Department of Mathematics \\ University of California \\ Santa Barbara, CA 93106-3080 \\ cooper@math.ucsb.edu \\ Jiang-Hua Lu \\ Department of Mathematics \\ The University of Hong Kong \\ Pokfulam Rd., Hong Kong \\ jhlu@maths.hku.hk \\ Paul Yang \\ Department of Mathematics \\ Princeton University \\ Princeton NJ 08544-1000 \\ yang@math.princeton.edu
}

These supporting institutions contribute to the cost of publication of this Journal, but they are not owners or publishers and have no responsibility for its contents or policies.

See inside back cover or msp.org/pjm for submission instructions.

The subscription price for 2014 is US $\$ 410 /$ year for the electronic version, and \$535/year for print and electronic.

Subscriptions, requests for back issues and changes of subscribers address should be sent to Pacific Journal of Mathematics, P.O. Box 4163, Berkeley, CA 94704-0163, U.S.A. The Pacific Journal of Mathematics is indexed by Mathematical Reviews, Zentralblatt MATH, PASCAL CNRS Index, Referativnyi Zhurnal, Current Mathematical Publications and Web of Knowledge (Science Citation Index).

The Pacific Journal of Mathematics (ISSN 0030-8730) at the University of California, c/o Department of Mathematics, 798 Evans Hall \#3840, Berkeley, CA 94720-3840, is published twelve times a year. Periodical rate postage paid at Berkeley, CA 94704, and additional mailing offices. POSTMASTER: send address changes to Pacific Journal of Mathematics, P.O. Box 4163, Berkeley, CA 94704-0163.

PJM peer review and production are managed by EditFLOW ${ }^{\circledR}$ from Mathematical Sciences Publishers.

\section{PUBLISHED BY}

\section{mathematical sciences publishers \\ nonprofit scientific publishing}

http://msp.org/

(C) 2014 Mathematical Sciences Publishers 


\section{PACIFIC JOURNAL OF MATHEMATICS}

Volume 269 No. $1 \quad$ May 2014

The asymptotic behavior of Palais-Smale sequences on manifolds with $\quad 1$ boundary

SÉRGIO AlMARAZ

The cup subalgebra of a $\mathrm{II}_{1}$ factor given by a subfactor planar algebra is maximal amenable

ARNAUD BROTHIER

Representation theory of type B and C standard Levi $W$-algebras

JONATHAN BROWN and SIMON M. GOODWIN

New invariants for complex manifolds and rational singularities

RONG DU and YUN GAO

Homogeneity groups of ends of open 3-manifolds

DENNIS J. GARITY and DUŠAN REPOVŠ

On the concircular curvature of a $(\kappa, \mu, \nu)$-manifold

Florence Gouli-Andreou and Evaggelia Moutafi

Genuses of cluster quivers of finite mutation type

FANG LI, JICHUN LIU and YICHAO YANG

Taut foliations in knot complements

TAO LI and RACHEL ROBERTS

On the set of maximal nilpotent supports of supercuspidal representations 169

QIN YUJUN

The natural filtrations of finite-dimensional modular Lie superalgebras of 199 Witt and Hamiltonian type

Keli Zheng, Yongzheng ZHANG and WeI Song

Free Brownian motion and free convolution semigroups: multiplicative 219 case

PING ZHONG 